\title{
Activation of tyrosine kinase c-Abl contributes to $\alpha$-synuclein-induced neurodegeneration
}

\author{
Saurav Brahmachari, ${ }^{1,2,3}$ Preston Ce, ${ }^{1,2,3}$ Su Hyun Lee, ${ }^{1,2}$ Donghoon Kim, ${ }^{1,2,4}$ Senthilkumar S. Karuppagounder, ${ }^{1,2,3}$ \\ Manoj Kumar, ${ }^{1,2,3}$ Xiaobo Mao,, 1,2,3 Joo Ho Shin, ${ }^{1,2,3}$ Yunjong Lee, ${ }^{1,2,3}$ Olga Pletnikova, ${ }^{5}$ Juan C. Troncoso, ${ }^{2,5}$ \\ Valina L. Dawson, ${ }^{1,2,3,6,7}$ Ted M. Dawson, ${ }^{1,2,3,7,8}$ and Han Seok Ko ${ }^{1,2,4}$ \\ 1Neuroregeneration and Stem Cell Programs, Institute for Cell Engineering, and 2Department of Neurology, The Johns Hopkins University School of Medicine, Baltimore, Maryland, USA \\ ${ }^{3}$ Adrienne Helis Malvin Medical Research Foundation, New Orleans, Louisiana, USA. ${ }^{4}$ Diana Helis Henry Medical Research Foundation, New Orleans, Louisiana, USA. ${ }^{5}$ Department of Pathology, \\ Division of Neuropathology, ${ }^{6}$ Department of Physiology, ${ }^{7}$ Solomon H. Snyder Department of Neuroscience, and ${ }^{8}$ Department of Pharmacology and Molecular Sciences, \\ The Johns Hopkins University School of Medicine, Baltimore, Maryland, USA.
}

\begin{abstract}
Aggregation of $\alpha$-synuclein contributes to the formation of Lewy bodies and neurites, the pathologic hallmarks of Parkinson disease (PD) and $\alpha$-synucleinopathies. Although a number of human mutations have been identified in familial PD, the mechanisms that promote $\alpha$-synuclein accumulation and toxicity are poorly understood. Here, we report that hyperactivity of the nonreceptor tyrosine kinase c-Abl critically regulates $\alpha$-synuclein-induced neuropathology. In mice expressing a human $\alpha$-synucleinopathy-associated mutation (hA53T $\alpha$-syn mice), deletion of the gene encoding c-Abl reduced $\alpha$-synuclein aggregation, neuropathology, and neurobehavioral deficits. Conversely, overexpression of constitutively active c-Abl in hA53T $\alpha$-syn mice accelerated $\alpha$-synuclein aggregation, neuropathology, and neurobehavioral deficits. Moreover, c-Abl activation led to an age-dependent increase in phosphotyrosine $39 \alpha$-synuclein. In human postmortem samples, there was an accumulation of phosphotyrosine $39 \alpha$-synuclein in brain tissues and Lewy bodies of PD patients compared with age-matched controls. Furthermore, in vitro studies show that c-Abl phosphorylation of $\alpha$-synuclein at tyrosine 39 enhances $\alpha$-synuclein aggregation. Taken together, this work establishes a critical role for c-Abl in $\alpha$-synuclein-induced neurodegeneration and demonstrates that selective inhibition of c-Abl may be neuroprotective. This study further indicates that phosphotyrosine 39 $\alpha$-synuclein is a potential disease indicator for PD and related $\alpha$-synucleinopathies.
\end{abstract}

\section{Introduction}

Parkinson disease (PD) is the second most common neurodegenerative disorder and is characterized by the preferential loss of dopamine (DA) neurons leading to motoric dysfunction including bradykinesia, rest tremor, rigidity, and postural instability $(1,2)$. Moreover, other regions of the nervous system are also affected, which contribute to the nonmotor symptoms including autonomic dysfunction, cognitive and neuropsychiatric disorders, and sleep disorders, among others (3). Neuropathologically, PD is characterized by the accumulation and aggregation of $\alpha$-synuclein, which contributes to the formation of Lewy bodies and Lewy neurites (4-6). Numerous studies indicate that aggregation and accumulation of $\alpha$-synuclein is intimately linked to the degenerative process (4-8). Mutations (A53T, A30P, and E46K) or duplication or triplication of WT $\alpha$-synuclein have been associated with rare forms of familial PD (9-12). Many $\alpha$-synuclein transgenic mouse models of the familial forms of PD due to mutations in $\alpha$-synuclein have been created $(7,13-17)$. These models replicate many of the features of $\alpha$-synucleinopathy-induced neurodegeneration that is present in human PD and diffuse Lewy body disease (7, 13-17). Posttranslational modifications of $\alpha$-synuclein such as nitrosylation, oxida-

Conflict of interest: The authors have declared that no conflict of interest exists. Submitted: November 23, 2015; Accepted: May 5, 2016.

Reference information: J Clin Invest. 2016;126(8):2970-2988. doi:10.1172/JCI85456. tion, and phosphorylation play a role in modulating $\alpha$-synuclein aggregation and toxicity (18-21).

c-Abl is a nonreceptor tyrosine kinase that is activated by oxidative and cellular stress (22). c-Abl is activated in human postmortem PD brain in the striatum and substantia nigra $(23,24)$. Recently, there have been a few reports indicating that c-Abl inhibition might be beneficial in PD and $\alpha$-synucleinopathies $(23,25$, 26). However, the lack of selectivity of the c-Abl inhibitors used in these studies has made it difficult to conclude whether c-Abl inhibition could be an effective neuroprotective strategy.

Here we report that c-Abl activation plays a significant and substantial role in the pathogenesis of the human A53T $\alpha$-synuclein transgenic model of neurodegeneration. In particular, expression of a constitutively active form of c-Abl (BCR-ABL) markedly exacerbates the pathology and shortens the lifespan of human A53T $\alpha$-synuclein transgenic mice $\left(\operatorname{PrP}-S N C A^{A 53 T}\right.$, herein referred to as hA53T $\alpha$-syn mice). Importantly, expression of BCR-ABL in WT mice leads to $\alpha$-synuclein pathology and loss of DA neurons. On the other hand, knockout of $c$ - $A b l$ markedly reduces the pathology and extends the lifespan of hA53T $\alpha$-syn transgenic mice. By this dual approach, we provide evidence that c-Abl activation significantly modulates $\alpha$-synuclein neurodegeneration. Moreover, c-Abl phosphorylates $\alpha$-synuclein on tyrosine 39, and phosphorylation of tyrosine 39 of $\alpha$-synuclein increases $\alpha$-synuclein's propensity to aggregate. Phosphorylation of tyrosine 39 of $\alpha$-synuclein 
correlates with disease pathogenesis and progression in hA53T $\alpha$ syn mice and is contained within Lewy bodies in human PD. These findings suggest that phosphotyrosine $39 \alpha$-synuclein might be a progression marker for PD and $\alpha$-synucleinopathies.

\section{Results}

$c$-Abl overactivation in $\alpha$-synuclein transgenic mice. To study the potential role of $\mathrm{c}$-Abl in $\alpha$-synuclein-induced neurodegeneration, the G2-3 human A53T $\alpha$-synuclein (hA53T $\alpha$-syn) transgenic model, in which human A53T $\alpha$-synuclein transgene is driven by the mouse prion promoter, was used (27). These mice exhibit a progressive neurodegenerative disorder that leads to premature lethality and exhibit substantial neurodegeneration accompanying typical $\alpha$-synuclein pathology including serine 129 phosphorylation, the formation of $\alpha$-synuclein fibrils, and markers of mitochondrial and oxidative damage (27-30). In symptomatic hA53T $\alpha$-syn transgenic mice, c-Abl activation was monitored via IB analysis of tyrosine 245 autophosphorylation of c-Abl (23, $24,26)$ in affected versus nonaffected CNS regions. A greater than twofold activation of $\mathrm{c}-\mathrm{Abl}$ is observed in areas of pathology including the spinal cord (Figure 1, A and B) and brain stem (Figure $1, \mathrm{C}$ and D) in hA53T $\alpha$-syn mice, whereas no activation is observed in the cortex, which is relatively unaffected (Figure 1, E and F). There is no c-Abl activation observed in the ventral midbrain as well, a region that lacks pathology in these mice (Supplemental Figure 9; supplemental material available online with this article; doi:10.1172/JCI85456DS1). Immunostaining of phospho-c-Abl colocalizes with $\alpha$-synuclein in the brain stem of the hA53T $\alpha$-syn mice (Figure $1 G$ ). To determine whether c-Abl activation is co-incident with pathology, immunostaining for $\alpha$-synuclein phosphorylated at serine 129 was performed. Phospho-c-Abl colocalizes with phosphoserine $129 \alpha$-synuclein in the brain stem of the hA53T $\alpha$ syn mice, suggesting that c-Abl activation occurs in the setting of $\alpha$-synuclein pathology (Figure $1 \mathrm{H})$. In the cortex, which is unaffected, there is no colocalization of phospho-c-Abl with $\alpha$-synuclein or phosphoserine $129 \alpha$-synuclein (Figure 1, G and H).

c-Abl overexpression exacerbates $\alpha$-synuclein pathologies. To test the role of c-Abl in $\alpha$-synuclein neurodegeneration, the hA53T $\alpha$ syn mice were cross-bred to the BCR-ABL Tet-Off transgenic mice [FVB/N-Tg(tetO-BCR/ABL1)2Dgt/J (Jax \#006202); referred to as $B C R-A B L$ mice]. BCR-ABL is a constitutively active form of c-Abl (22). Since human A53T $\alpha$-synuclein is driven by the mouse prion promoter (PrP), the PrP-tTA driver was used to drive BCR-ABL expression. $B C R-A B L$ hA53T $\alpha$-syn bigenic mice were created and cross-bred to the PrP-tTA driver mice. From this cross-breeding, littermates with the following genotypes were separated and aged: nontransgenic, $B C R-A B L$ PrP-tTA transgenic, hA53T $\alpha$-syn transgenic, $\operatorname{Pr} P$-tTA hA53T $\alpha$-syn transgenic, and BCR-ABL hA53T $\alpha$-syn PrP-tTA mice. In addition, another cohort of the trigenic, $B C R$ $A B L$ hA53T $\alpha$-syn PrP-tTA mice were treated with doxycycline to suppress $B C R-A B L$ expression (Figure $2 \mathrm{~A}$ ). The different genotype cohorts were aged, and survival was monitored. As previously described, hA53T $\alpha$-syn mice live an average of 10 months (27); 1 mouse lived to 18 months of age (Figure $2 \mathrm{~B}$ ). The nontransgenic mice and the $B C R-A B L \operatorname{Pr} P-t T A$ live a normal lifespan. According to previous reports, the tetracycline-controlled transactivator ( $t T A)$ may have mild toxicity, but its expression level usually remains under the threshold, and thus it does not cause any detrimental effects on mice (31). The PrP-tTA hA53T $\alpha$-syn mice live an average of 9.4 months with no significant difference in lifespan compared with hA53T $\alpha$-syn mice (Figure 2B). The BCR-ABL hA53T $\alpha$-syn PrP-tTA mice live an average of 6.5 months, and doxycycline restores the survival of $B C R-A B L$ hA53T $\alpha$-syn $\operatorname{PrP}$ - $t$ TA mice to the extent of the lifespan of the hA53T $\alpha$-syn mice (Figure 2B).

The different cohorts of mice were assessed for expression levels of BCR-ABL, phospho-c-Abl, and $\alpha$-synuclein at 3 months of age in brain stem, spinal cord, and cortex (Figure 2, C-H). Equivalent levels of $\alpha$-synuclein are overexpressed in the hA53T $\alpha$-syn mice compared with the $B C R-A B L$ hA53T $\alpha$-syn PrP-tTA mice, and doxycycline has no effect on the levels of $\alpha$-synuclein (Figure 2, C-H). Increased phospho-c-Abl is observed in the $B C R-A B L \operatorname{PrP}-t T A$ mice and the $B C R-A B L$ hA53T $\alpha$-syn $\operatorname{PrP}-t T A$ mice, which is suppressed by doxycycline in the $B C R-A B L$ hA53T $\alpha$-syn $\operatorname{Pr} P$ - $t$ TA mice (Figure 2, C-H). Interestingly, the levels of BCR-ABL are increased in the $B C R-A B L$ hA53T $\alpha$-syn $\operatorname{PrP}$ - $t$ TA mice (Figure 2, C-H).

At 2 months of age there is no significant difference in body weight between the different genotype cohorts, but at 6 months of age when the animals are symptomatic the $B C R-A B L \mathrm{hA53T} \alpha-$ syn $\operatorname{PrP}$-tTA mice exhibit a significant reduction in body weight by approximately $20 \%$, that is prevented by doxycycline administration (Supplemental Figure 1A). Behavior was assessed over time (Supplemental Figure 1, B and C). According to previous reports, hA53T $\alpha$-syn mice show increased horizontal and vertical activities at preclinical ages (from 5 months onward to 5 days before onset of symptoms) compared with nontransgenic mice when examined under open field $(27,32)$. Increased hyperactivity indicates increased susceptibility of hA53T $\alpha$-syn mice to neurodegeneration. Therefore we wondered whether $B C R-A B L$ hA53T $\alpha$-syn $\operatorname{Pr} P$ - $t$ TA mice have any altered activities. Open-field monitoring reveals no significant difference in vertical activity among the genotypes at 4 months of age, but at 5 months of age it is increased in BCR-ABL hA53T $\alpha$-syn PrP-tTA compared with the nontransgenic mice (Supplemental Figure 1C). Horizontal activity is increased at 5 months of age in the hA53T $\alpha$-syn and increased at 4 and 5 months of age in the BCR-ABL hA53T $\alpha$-syn $\operatorname{PrP}$-tTA mice, which is significantly greater than that observed in the hA53T $\alpha$-syn mice (Supplemental Figure 1B). Doxycycline prevents the change in horizontal activity (Supplemental Figure 1B). These data suggest that BCR-ABL hA53T $\alpha$-syn PrP-tTA mice might be at higher risk of neurodegeneration. As deficits in rotarod activities are linked to early defects in motor neurons (27), we assessed rotarod performance for all different genotypes. There is no difference in an accelerating rotarod paradigm at 3 and 5 months of age between the different genotypes (S. Brahmachari, unpublished observations).

In symptomatic $B C R-A B L$ hA53T $\alpha$-syn $\operatorname{PrP}$-tTA mice at approximately 6 months of age, there is substantial neuronal accumulation of human phosphoserine $129 \alpha$-synuclein in a number of brain regions, including the brain stem and cerebellum (Figure 3A). Since neuronal accumulation of ubiquitin is a prominent indicator of pathology, ubiquitin immunoreactivity was examined. There is a prominent accumulation of ubiquitin in neurites and cell bodies in the brain stem and cerebellum (Figure 3B). Glial fibrillary acidic protein (GFAP) was analyzed as an indirect indication of overall 
A

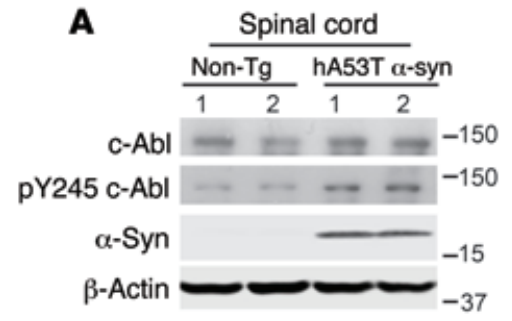

B

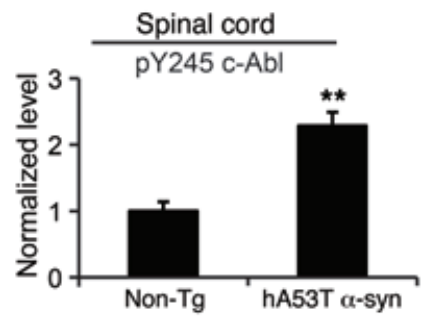

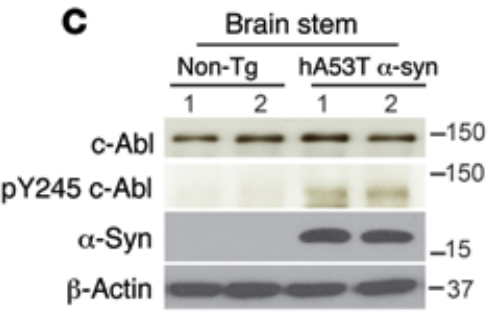

D

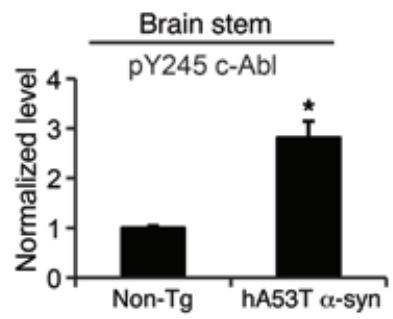

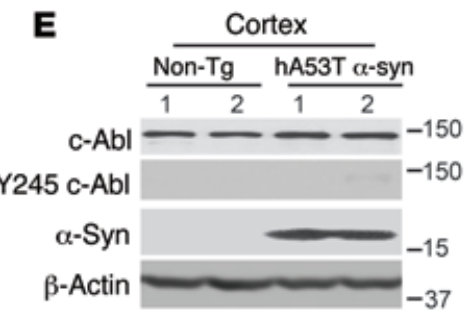

$\mathbf{F}$

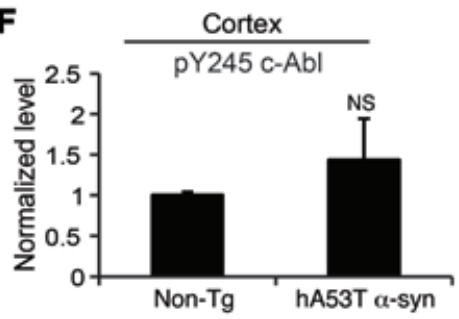

G
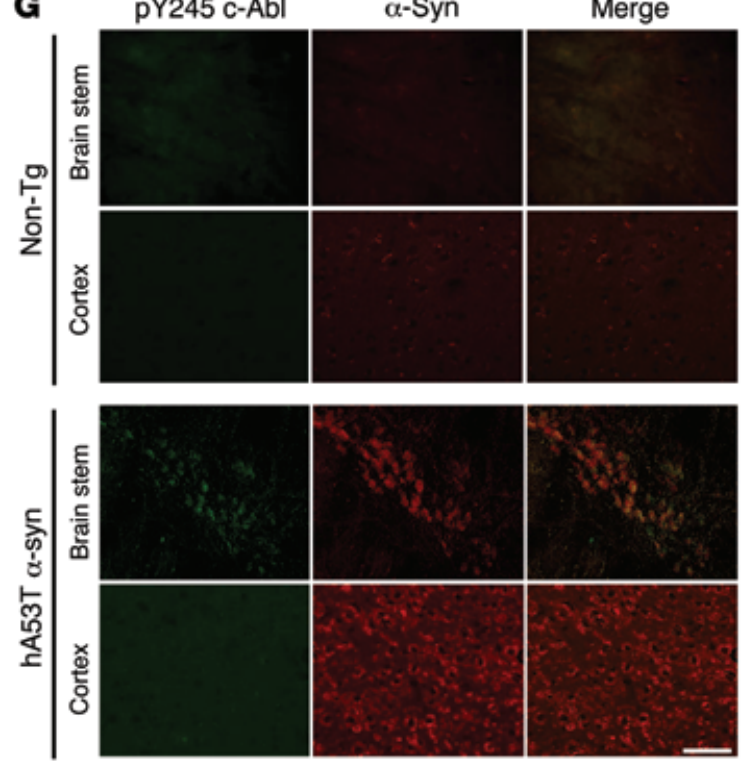

H
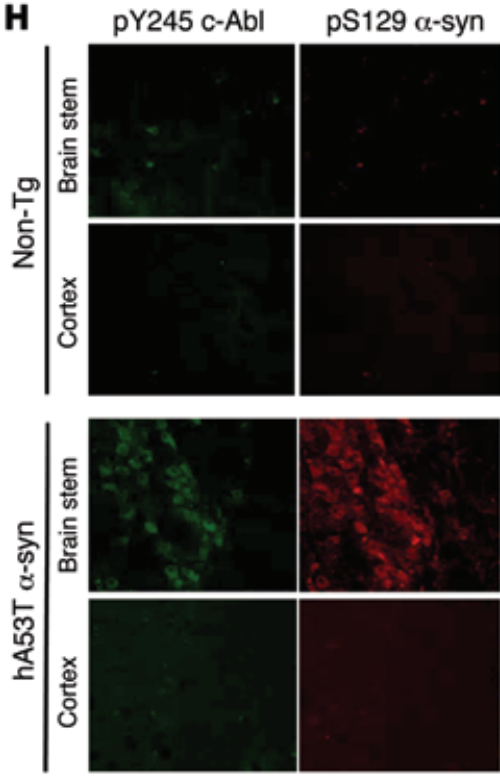

Merge

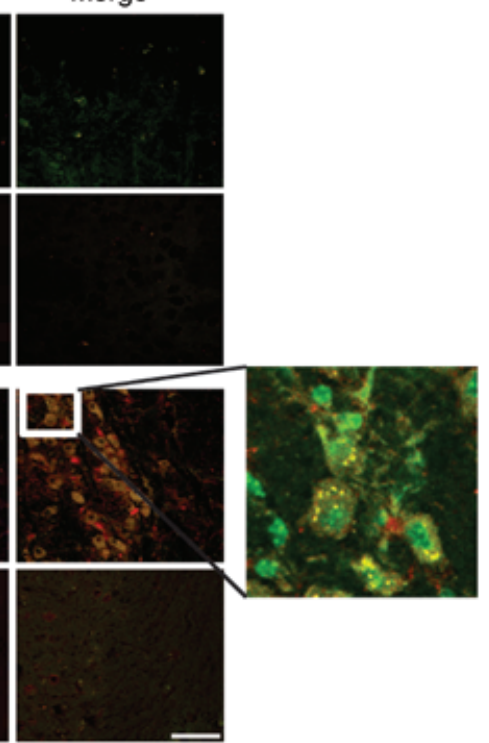

Figure 1. c-Abl is overactivated in symptomatic hA53T $\alpha$-syn transgenic mice. (A, C, and E) Representative immunoblots of c-Abl, pY245 c-Abl, $\alpha$-synuclein ( $\alpha$-syn), and $\beta$-actin in the spinal cord, brain stem, and cortex from symptomatic hA53T $\alpha$-syn transgenic mice and age-matched nontransgenic (Non-Tg) littermate controls. (B, D, and F) Quantification of pY245 c-Abl protein levels normalized to c-Abl ( $n=4$ per group). Data are from 3 independent experiments. Statistical significance was determined by 2 -tailed unpaired Student's $t$ test. Quantified data are expressed as the mean \pm SEM. ${ }^{*} P<0.05$, ${ }^{*} P<0.01$. (G) Representative immunofluorescent images of pY245 c-Abl (green) and $\alpha$-syn (red) in the brain stem and cortex from symptomatic hA53T $\alpha$-syn transgenic mice and age-matched nontransgenic littermate controls ( $n=3$ per group). Scale bar: $50 \mu m$. (H) Representative confocal images of pY245 c-Abl (green) and pS129 $\alpha$-syn (red) in the brain stem and cortex from symptomatic hA53T $\alpha$-syn transgenic mice and age-matched nontransgenic littermate controls ( $n=3$ per group). Enlarged image (zoom-in, $\times 35$; original magnification, $\times 40$ ) at right shows colocalization of pY245 c-Abl and pS129 $\alpha-5 y n$. Scale bar: $50 \mu$ m.

neurodegeneration, and there is substantial GFAP immunoreactivity in the brain stem and cerebellum of $B C R-A B L$ hA53T $\alpha$-syn PrP-tTA mice (Figure 3C). At 6 months of age there is no significant accumulation of phosphoserine $129 \alpha$-synuclein, ubiquitin, or GFAP in any of the other mouse genotypes (Figure 3, A-C). The accumulation of detergent-insoluble A53T $\alpha$-synuclein species in biochemical fractionation assays is associated with pathologic neuronal accumulation of $\alpha$-synuclein in transgenic mice $(27,30)$; thus the brain stem of the different genotypes was fractionated into detergent-soluble and -insoluble fractions (Figure 3, D-G). Immunoblotting of these fractions demonstrates that in the brain stem of $B C R-A B L$ hA53T $\alpha$-syn PrP-tTA mice there is accumulation of insoluble phosphoserine $129 \alpha$-synuclein and high-molecular weight species of $\alpha$-synuclein, as well as proteolytic fragments of $\alpha$-synuclein. Doxycycline treatment in the BCR-ABL hA53T $\alpha$-syn $\operatorname{Pr} P$-tTA mice prevents the accumulation of insoluble high-molecular weight species of $\alpha$-synuclein, monomeric $\alpha$-synuclein, and insoluble phosphoserine $129 \alpha$-synuclein (Figure 3, F and G). No abnormalities are observed in the other genotypes. Mild accumulation of soluble high-molecular weight species of $\alpha$-synuclein as well as phosphoserine $129 \alpha$-synuclein is detected in hA53T $\alpha$-syn and $B C R-A B L$ hA53T $\alpha$-syn $\operatorname{PrP}-t T A$ mice with or without doxycycline, but no significant difference in their levels is observed among them (Figure 3, D and E). Taken together, these results 
A

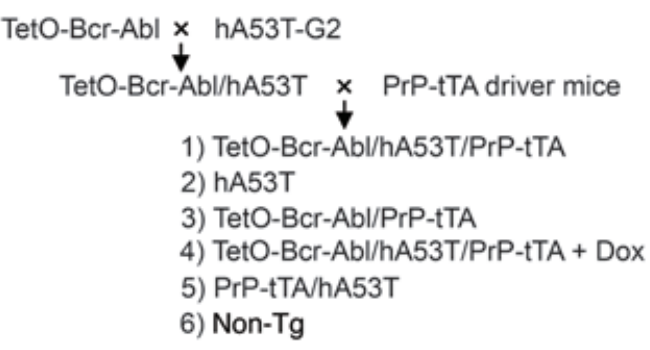

B

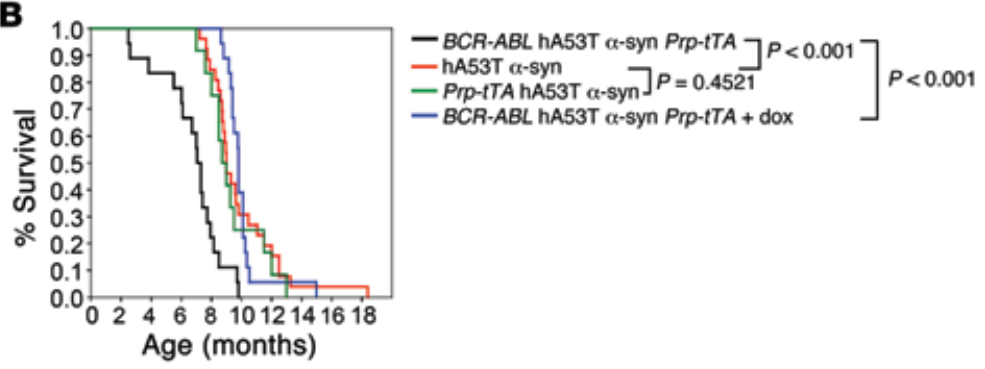

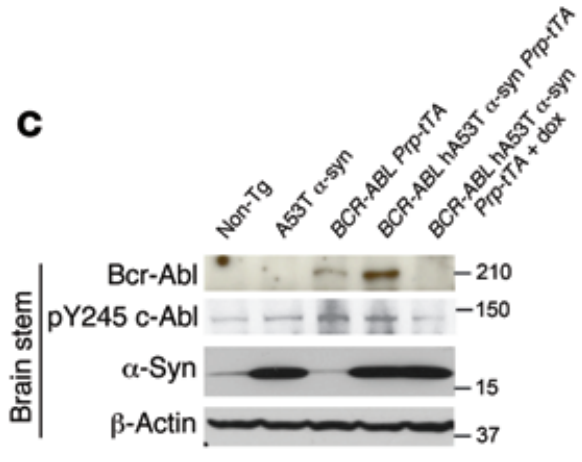

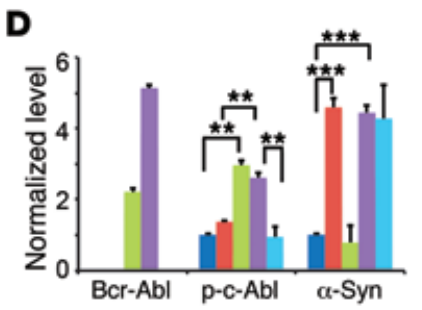

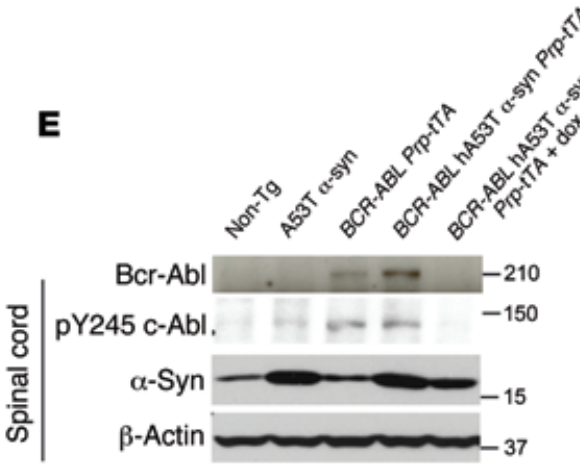
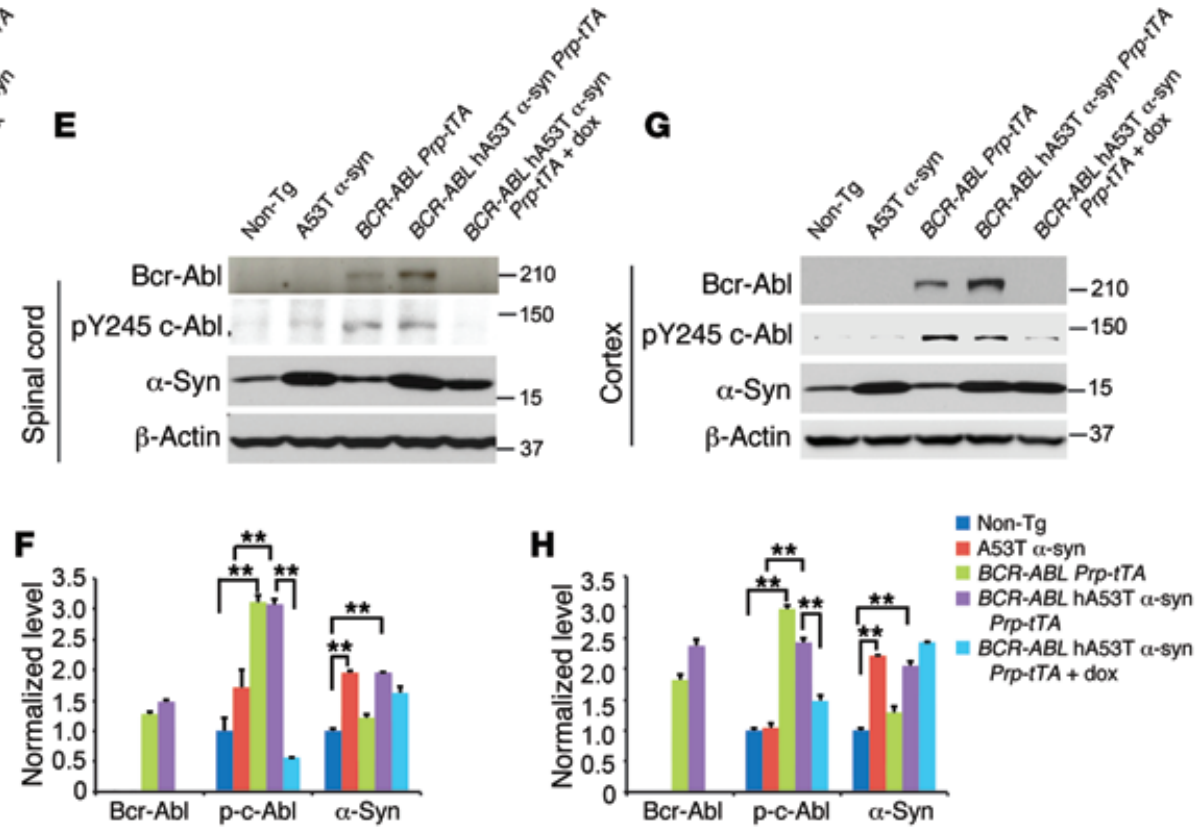

Figure 2. c-Abl overexpression decreases survival of hA53T $\alpha$-syn transgenic mice. (A) Breeding strategy to generate $B C R-A B L$ hA53T $\alpha$-syn $P r P-t T A$ trigenic mice. (B) Kaplan-Meier survival curve analysis for hA53T $\alpha$-syn, PrP-tTA hA53T $\alpha$-syn, BCR-ABL hA53T $\alpha$-syn PrP-tTA trigenic, and BCR-ABL hA53T $\alpha$ syn PrP-tTA trigenic mice with doxycycline ( $n=20-30$ mice per group). Statistical analysis was performed by Mann-Whitney-Wilcoxon test. (C, E, and $\mathbf{G})$ Representative immunoblots of BCR-ABL, pY245 c-Abl, $\alpha$-syn, and $\beta$-actin in the brain stem, spinal cord, and cortex from 3-month-old BCR-ABL hA53T $\alpha$ syn PrP-tTA trigenic mice with or without doxycycline and age-matched littermate controls. (D, F, and $\mathbf{H}$ ) Quantification of BCR-ABL, pY245 c-Abl, and $\alpha$-syn protein levels normalized to $\beta$-actin ( $n=3$ per group). Data are from 3 independent experiments. Statistical significance was determined by 1 -way ANOVA with Tukey's post-test of multiple comparisons. Quantified data are expressed as the mean $\pm \mathrm{SEM} .{ }^{* *} P<0.01,{ }^{* * *} P<0.001$.

indicate that c-Abl activity is capable of accelerating A53T $\alpha$-synuclein-induced neuropathology and neurobehavioral deficits.

c-Abl overexpression in WT mice leads to DA neuron degeneration and WT $\alpha$-synuclein pathologies. BCR-ABL PrP-tTA transgenic mice were aged and examined. At 2 years of age there is significant loss of DA neurons as assessed by quantitative unbiased stereology with a $30 \%$ reduction in tyrosine hydroxylase $\mathrm{(TH}$ ) immunoreactivity and Nissl staining (Supplemental Figure 2, A and B). Accompanying the reduction in DA neurons there is a significant reduction in striatal TH fiber density (Supplemental Figure 2, C and D). These results suggest that overactivation of c-Abl is sufficient to cause degeneration of DA neurons. To determine whether the loss of DA neurons is selective, we examined the cortex, hippocampus, cerebellum, locus coeruleus (LC), and nucleus basalis. There are no discernible abnormalities of the cortex, hippocampus, and cerebellum (Supplemental Figure 2E). In addition, stereologic counts of cortical neurons reveal no significant difference between BCR-ABL PrP-tTA mice and age-matched nontransgenic mice (Supplemental Figure $2 \mathrm{~F}$ ), and there is a trend toward a loss of nucleus basalis neurons
(S. Brahmachari, unpublished observations). Interestingly, there is a marked decrease of TH immunoreactivity in the $\mathrm{LC}$ region as evident from a 50\% reduction in TH-positive as well as Nissl-positive neurons (Supplemental Figure 2, G and H). The loss in TH immunoreactivity indicates degeneration of norepinephrine (NE) neurons in the LC. This finding is consistent with previous evidence that NE neurons in the LC undergo degeneration, which often precedes DA neuronal loss in the substantia nigra pars compacta (SNPc) $(33,34)$. Future studies are needed to evaluate whether LC neurons undergo early degeneration in this model, prior to the loss of DA neurons in the SNPc. Furthermore, the IB analysis in the ventral midbrain region of these mice shows substantial increase in the levels of activated c-Abl (BCR-ABL and pY245 c-Abl), phosphotyrosine 39 $\alpha$-synuclein (see below), and phosphoserine $129 \alpha$-synuclein along with accumulation of high-molecular weight species of $\alpha$-synuclein (Supplemental Figure 6, I and J).

$c$-Abl deletion reduces $\alpha$-synuclein pathologies. To directly test the role of c-Abl in $\alpha$-synuclein pathology, the hA53T $\alpha$-syn mice were cross-bred to conditional c-Abl knockout mice. Since germ- 

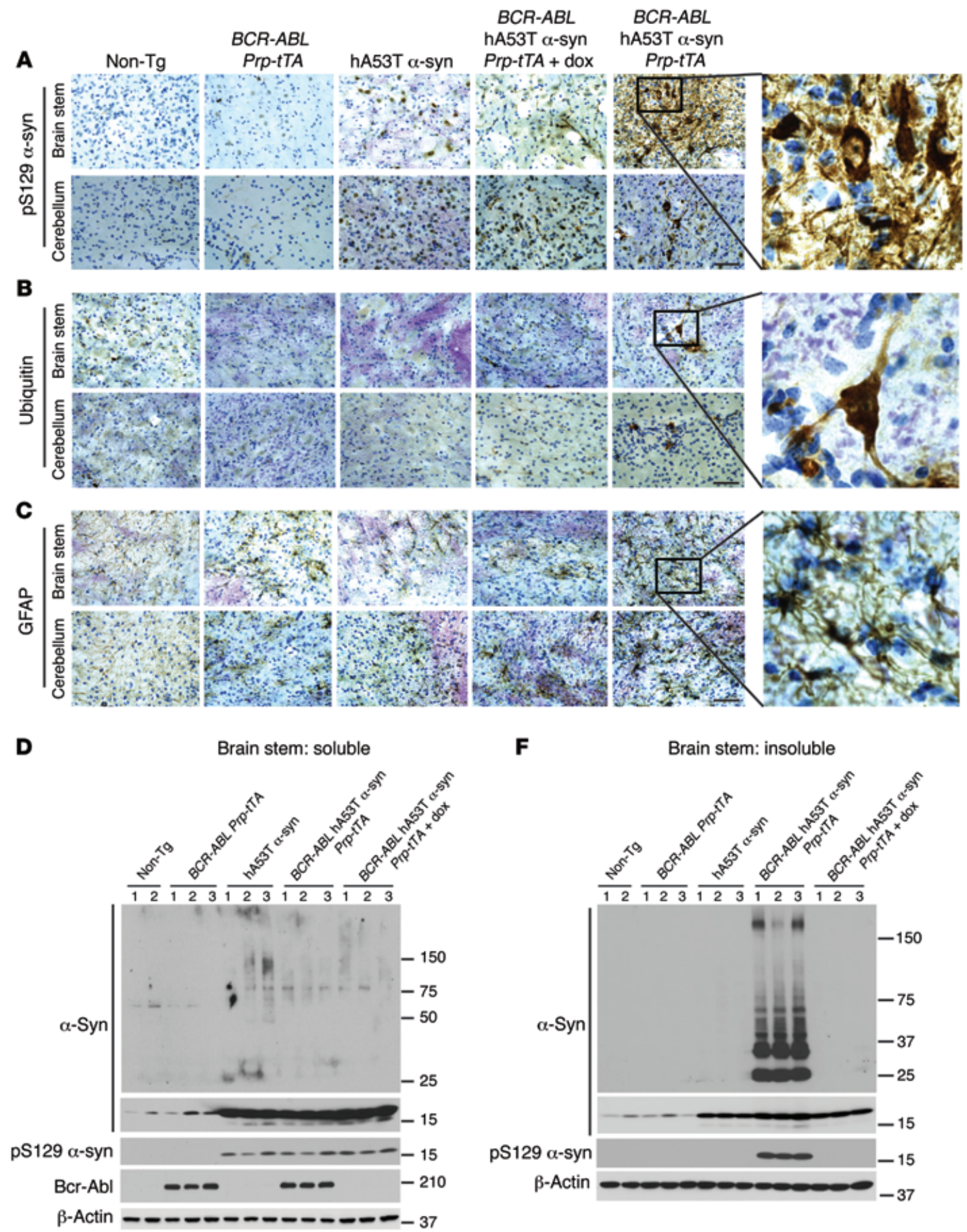

$\mathbf{F}$

Brain stem: insoluble

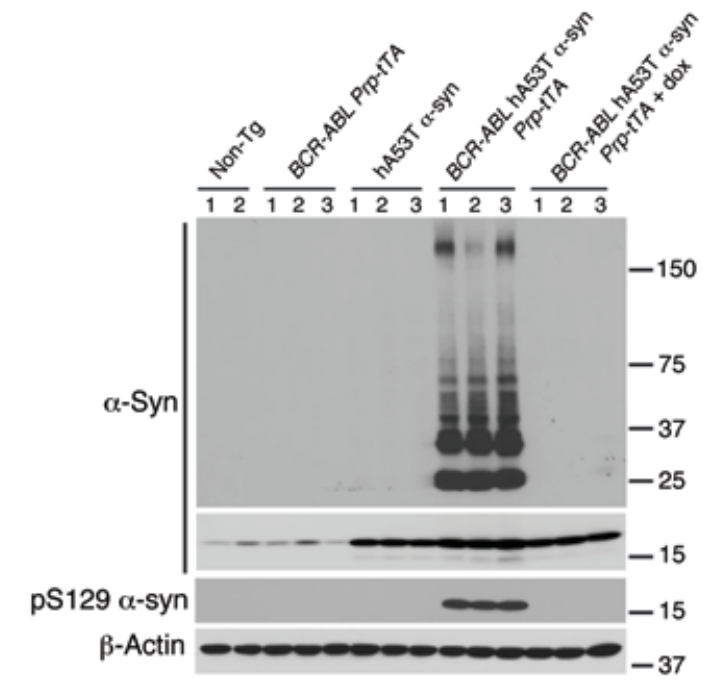

E
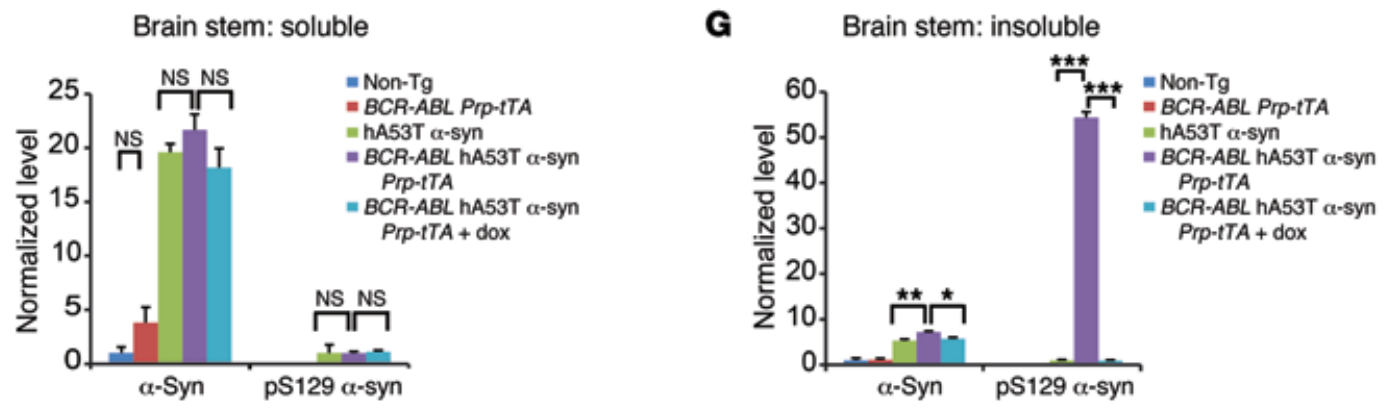
Figure 3. c-Abl overexpression exacerbates $\alpha$-synuclein pathologies. (A-C) Representative pS129 $\alpha$-syn, ubiquitin, and GFAP IHC in the brain stem and cerebellum of 6-month-old symptomatic BCR-ABL hA53T $\alpha$-syn PrP-tTA trigenic mice with or without doxycycline and age-matched littermate controls ( $n=3$ per group). Enlarged images (zoom-in, $\times 30$; original magnification, $\times 40$ ) of the indicated regions are shown at right. Scale bars: $50 \mu \mathrm{m}$. (D) Representative immunoblots of $\alpha$-syn, pS129 $\alpha$-syn, BCR-ABL, and $\beta$-actin in the detergent-soluble fraction of brain stem from 6 -monthold symptomatic $B C R$-ABL hA53T $\alpha$-syn PrP-tTA trigenic mice with or without doxycycline and age-matched littermate controls. (E) Quantification of $\alpha$-syn monomer and pS129 $\alpha$-syn protein levels in $\mathbf{D}$ normalized to $\beta$-actin and $\alpha$-syn monomer, respectively ( $n=5$ mice per group). (F) Representative immunoblots of $\alpha$-syn, pS129 $\alpha$-syn, and $\beta$-actin in the detergent-insoluble fraction of brain stem from 6-month-old symptomatic BCR-ABL hA53T $\alpha$ syn PrP-tTA trigenic mice with or without doxycycline and age-matched littermate controls. (G) Quantification of $\alpha$-syn monomer and pS129 $\alpha$-syn protein levels in $\mathbf{F}$ normalized to $\beta$-actin and $\alpha$-syn monomer, respectively ( $n=5$ mice per group). (D-C) Data are from 3 independent experiments. Statistical significance was determined by 1-way ANOVA with Tukey's post-test of multiple comparisons. Quantified data are expressed as the mean \pm SEM. ${ }^{*} P<0.05,{ }^{* *} P<0.01,{ }^{* *} P<0.001$

line c-Abl knockout mice are embryonic lethal $(35,36)$, conditional c-Abl knockout mice were used in which $c-A b l^{f / f l}$ were cross-bred to Nestin-Cre driver mice to selectively delete c-Abl from neurons as previously described $(23,37) \cdot c-A b l^{f / f l}$ mice were cross-bred to the hA53T $\alpha$-syn mice to create $c-A b l^{t / f l}$ hA53T $\alpha$-syn transgenic mice, which were then cross-bred to $c-A b l^{f l-}$ Nestin-Cre ${ }^{+}$mice. From this cross-breeding, littermates with the following genotypes were separated and aged: $c$-Ablt/- hA53T $\alpha$-syn Nestin-Cre ${ }^{+}$(referred to as $c$ - $A b l^{K O}$ hA53T $\alpha$-syn), $c$ - $A b l^{f l / f l}$ hA53T $\alpha$-syn (referred to as $c-A b l^{W T}$ hA53T $\alpha$-syn), $c$-Abl $l^{f l-}$ Nestin-Cre $e^{+}$(referred to as $c-A b l^{K O}$ ), and $c-A b l^{f / f l}$ (referred to as $c-A b l^{W T}$ ) (Figure $4 \mathrm{~A}$ ). The different genotype cohorts were aged, and survival was monitored. As previously described, hA53T $\alpha$-syn mice live an average of 10 months (27). Knockout of $c-A b l$ significantly prolongs the survival of the hA53T $\alpha$-syn mice by 3 months (Figure 4B). The different cohorts of mice were assessed for expression levels of c-Abl and $\alpha$-synuclein at 2 months of age in brain stem, spinal cord, and cortex (Figure $4, \mathrm{C}-\mathrm{H})$. Equivalent levels of $\alpha$-synuclein are expressed among the different genotypes (Figure $4, \mathrm{C}-\mathrm{H}$ ). The different cohorts of mice were also assessed for c-Abl by immunofluorescence at 9 months of age in the brain stem (Supplemental Figure 8, A and B). At 5 months of age when the mice are asymptomatic, there is no weight loss in the hA53T $\alpha$-syn mice. There is an approximately $20 \%$ loss of weight in the $c-A b l^{K O}$ mice that is not exacerbated by the coexpression of hA53T $\alpha$-syn (Supplemental Figure 3A). At 9 months of age there is a significant weight loss in hA53T $\alpha$-syn mice, with no further loss of weight in the $c-A b l^{K O}$ or the $c-A b l^{K O}$ hA53T $\alpha$ syn mice (Supplemental Figure 3A). Behavior was assessed over time (Supplemental Figure 3, B and C). Open-field monitoring reveals no significance in vertical and horizontal activity among the genotypes at 4 months of age (S. Brahmachari, unpublished observations). At 6 months of age there is a significant increase in horizontal and vertical activity in hA53T $\alpha$-syn mice as previously described (32) (Supplemental Figure 3, B and C), which is significantly reduced in the $c-A b l^{K O} \mathrm{hA53T} \alpha$-syn mice (Supplemental Figure 3, B and C). These results suggest that $c-A b l^{K O}$ hA53T $\alpha$-syn mice exhibit delayed neurodegeneration.
In symptomatic hA53T $\alpha$-syn mice at approximately 9 months of age, there is substantial neuronal accumulation of human phosphoserine $129 \alpha$-synuclein in a number of brain regions, including the brain stem and cerebellum (Figure 5A). There is a prominent accumulation of ubiquitin in neurites and cell bodies in all affected regions (Figure $5 \mathrm{~B}$ ). There is substantial GFAP immunoreactivity in the hA53T $\alpha$-syn mice (Figure 5C). At 9 months of age there is no significant accumulation of phosphoserine $129 \alpha$-synuclein, ubiquitin, or GFAP in any of the other mouse genotypes (Figure $5, \mathrm{~A}-\mathrm{C}$ ). The brain stem of the different genotypes was fractionated into detergent-soluble and -insoluble fractions (Figure 5, D-G). Immunoblotting of these fractions demonstrates that in the hA53T $\alpha$-syn mice there is accumulation of insoluble phosphoserine $129 \alpha$-synuclein and high-molecular weight species of $\alpha$-synuclein. However, no abnormalities are observed in the other genotypes (Figure 5, F and G). Mild accumulation of soluble phosphoserine $129 \alpha$-synuclein and high-molecular weight species of $\alpha$-synuclein as well as proteolytic fragments of $\alpha$-synuclein is detected in hA53T $\alpha$-syn and $c$ - $A b l^{K O}$ hA53T $\alpha$-syn mice, but no significant difference in their levels is observed among them (Figure 5, D and E).

$c$-Abl phosphorylates $\alpha$-synuclein. Since the transgenic c-Abl gain- and loss-of-function studies suggest that c-Abl activation plays a role in the pathogenesis of $\alpha$-synucleinopathies, experiments were performed to determine whether $\alpha$-synuclein interacts with and is phosphorylated by c-Abl. To determine whether c-Abl interacts with $\alpha$-synuclein, cotransfection experiments in SH-SY5Y cells were performed. GFP-tagged c-Abl (GFP-c-Abl) coimmunoprecipitates myc-tagged $\alpha$-synuclein (myc- $\alpha$-syn) (Figure $6 \mathrm{~A}$ ). Interestingly, a kinase-dead (KD) version (lysine 290 arginine) of c-Abl fails to interact with $\alpha$-synuclein. IP of $\alpha$-synuclein from mouse brain coimmunoprecipitates c-Abl, indicating that $\mathrm{c}-\mathrm{Abl}$ and $\alpha$-synuclein interact in vivo. $\mathrm{c}$-Abl fails to coimmunoprecipitate with mouse $\operatorname{IgG}$, which indicates that the interaction between $\mathrm{c}$-Abl and $\alpha$-synuclein is specific (Figure $6 \mathrm{~B}$ ). To further determine the specificity of the interaction, IP was compared between WT, $c$-Abl ${ }^{K O}$, hA53T $\alpha$-syn, and $c-A b l^{K O}$ hA53T $\alpha$-syn mice. Antibodies to $\alpha$-synuclein coimmunoprecipitate c-Abl from WT and hA53T $\alpha$-syn but not $c-A b l^{K O}$ nor $c-A b l^{K O}$ hA53T $\alpha$-syn mice (Figure $6 \mathrm{C}$ ).

An in vitro kinase assay was performed using recombinant c-Abl and recombinant $\alpha$-synuclein to determine whether c-Abl is competent to phosphorylate $\alpha$-synuclein. Phosphorylation was monitored with ${ }^{32} \mathrm{P}$-ATP. c-Abl phosphorylates $\alpha$-synuclein, and the c-Abl family kinase inhibitor STI-571 prevents the phosphorylation of $\alpha$-synuclein by c-Abl (Figure 6D). To determine the site of phosphorylation of $\alpha$-synuclein by c-Abl, 2-dimensional gel electrophoresis (2-DE) gel electrophoresis was used to separate phosphorylated from nonphosphorylated $\alpha$-synuclein (Figure 6E). c-Abl-phosphorylated $\alpha$-synuclein was submitted for mass spectrometry for the determination of the phosphorylation site. Mass spectrometric analysis provided $100 \%$ sequence coverage of $\alpha$-synuclein, and all tyrosine residues were investigated for phosphorylation status. Only tyrosine 39 is phosphorylated, whereas none of the other tyrosines were phosphorylated (Figure 6E). To confirm that tyrosine 39 was the sole site of phosphorylation by c-Abl, tyrosine 39 in $\alpha$-synuclein, as well as tyrosine 125, 133, and 136 , was mutated to phenylalanine. Cotransfection experiments in SH-SY5Y indicate that WT $\alpha$-synuclein is phosphorylated by c-Abl 
A

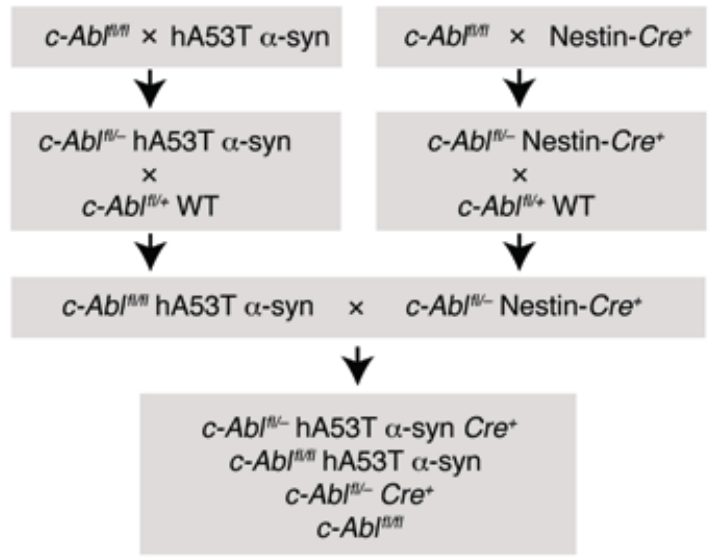

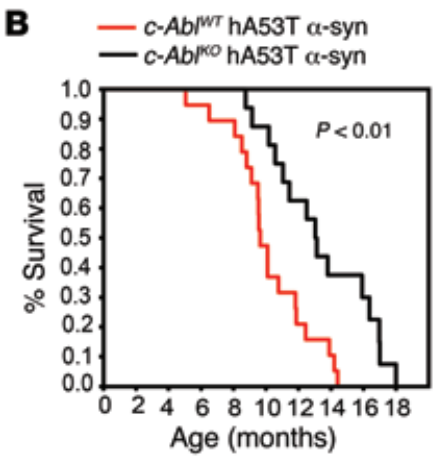

C
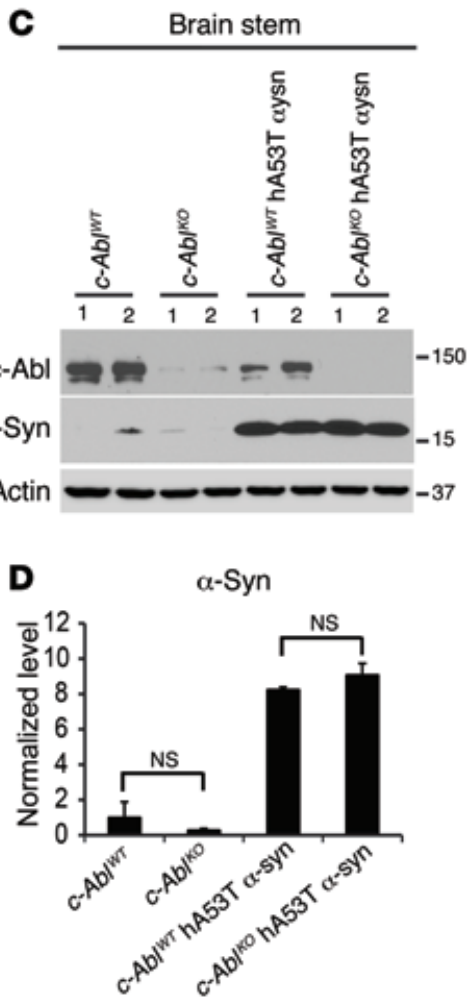

E
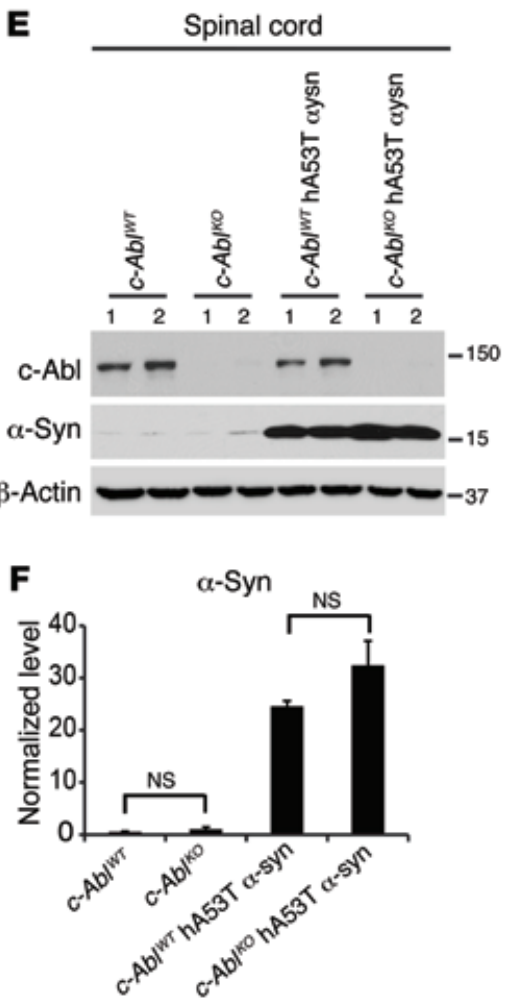

G
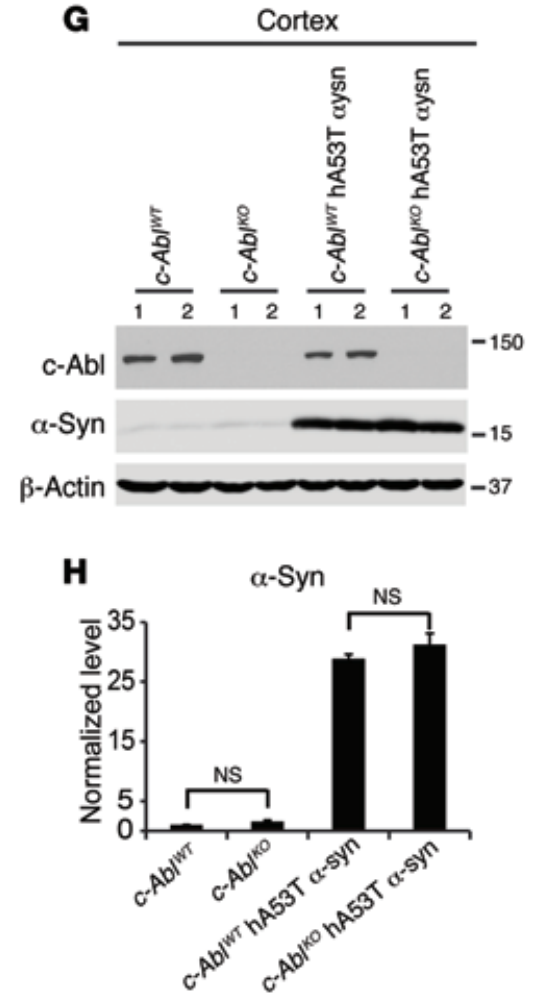

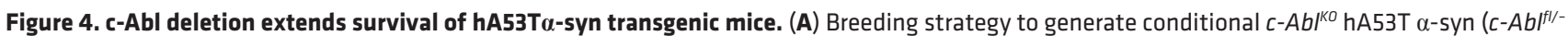
hA53T $\alpha$-syn Nestin-Cre) mice. (B) Kaplan-Meier survival curve analysis for $c-A b /{ }^{W T}$ hA53T $\alpha$-syn and $c-A b /{ }^{K o}$ hA53T $\alpha$-syn mice ( $n=20-30$ mice per group). Statistical analysis was performed by Mann-Whitney-Wilcoxon test. $P<0.01$. (C, E, and $\mathbf{G}$ ) Representative immunoblots of c-Abl, $\alpha$-syn, and $\beta$-actin in the brain stem, spinal cord, and cortex from 2-month-old c-Ab/Ko hA53T $\alpha$-syn mice and age-matched littermate controls. (D, F, and $\mathbf{H})$ Quantification of $\alpha$-syn protein levels normalized to $\beta$-actin ( $n=3$ per group). Data are from 3 independent experiments. Statistical significance was determined by 1 -way ANOVA with Tukey's post-test of multiple comparisons. Quantified data are expressed as the mean \pm SEM.

but not c-Abl-KD. c-Abl is not able to phosphorylate $\alpha$-synuclein $\mathrm{Y} 39 \mathrm{~F}$, but is able to tyrosine-phosphorylate $\alpha$-synuclein $\mathrm{Y} 125 \mathrm{~F}$, Y133F, and Y136F (Figure 6F). These results taken together indicate that c-Abl exclusively phosphorylates $\alpha$-synuclein on tyrosine 39. To further explore phosphorylation of $\alpha$-synuclein on tyrosine 39 , a phospho-specific antibody was generated and confirmed to specifically recognize phosphotyrosine 39 on $\alpha$-synuclein (Figure $6 \mathrm{~F}$ and Supplemental Figure 4A).

The levels of phosphotyrosine $39 \alpha$-synuclein were monitored in the brain stem and cortex of the hA53T $\alpha$-syn mice and compared with phosphoserine 129 at different ages by biochemical fractionation assays (Figure 7 and Supplemental Figure 4, B-E). As previously reported, phosphoserine $129 \alpha$-synuclein is increased in the brain stem of hA53T $\alpha$-syn mice and is completely absent from the nontransgenic controls $(29,38)$ (Figure 7). The levels of phosphoserine $129 \alpha$-synuclein in the detergent-soluble fractions of the brain stem do not change during the lifespan of the hA53T $\alpha$ syn mice (Figure 7, C and D). In contrast, phosphotyrosine $39 \alpha$-synuclein is detected at 2 months of age and increases significantly in asymptomatic (4 and 6 months) hA53T $\alpha$-syn mice and then decreases, but it still remains significantly increased in symptomatic hA53T $\alpha$-syn mice compared with 2 months of age (Figure 7, C 
and D). However, in the detergent-insoluble fractions, the level of phosphotyrosine $39 \alpha$-synuclein is mildly to moderately detected, and phosphoserine $129 \alpha$-synuclein is barely detectable in asymptomatic hA53T $\alpha$-syn mice at 2, 4, and 6 months of age, but their levels dramatically and significantly increase in the brain stem of early-symptomatic mice at 7-8 months of age and increase further in late-stage symptomatic mice at 9-12 months of age, when high-molecular weight species of $\alpha$-synuclein are observed (Figure 7, E-G). Accompanying the increase in phosphotyrosine 39 $\alpha$-synuclein is an increase in phospho-c-Abl (Figure 7, C-F). Similar results are observed in the spinal cord (S. Brahmachari, unpublished observations). Interestingly, biochemical fractionation assays in the cortex region of hA53T $\alpha$-syn mice indicate that there is a significant increase in phosphotyrosine $39 \alpha$-synuclein level in the detergent-soluble fractions of asymptomatic (6 months) but not of symptomatic hA53T $\alpha$-syn mice, which is also associated with mild accumulation of high-molecular weight species of $\alpha$-synuclein without any noticeable detection of phosphoserine $129 \alpha$-synuclein (Supplemental Figure 4, B and C). In contrast, no phosphotyrosine $39 \alpha$-synuclein or phosphoserine $129 \alpha$-synuclein is detected in the detergent-insoluble fractions (Supplemental Figure 4, D and E). Immunostaining for phosphotyrosine $39 \alpha$-synuclein demonstrates that phosphotyrosine $39 \alpha$-synuclein accumulates in affected brain regions of symptomatic hA53T $\alpha$-syn mice including brain stem and cerebellum, but not in unaffected brain regions such as the cortex (Supplemental Figure 5, A, B, and D). Phosphotyrosine $39 \alpha$-synuclein levels are increased in the BCR$A B L$ hA53T $\alpha$-syn PrP-tTA mice (Supplemental Figure 5C), and they are markedly reduced in the $c-A b l^{K O}$ hA53T $\alpha$-syn as assessed by immunostaining (Supplemental Figure 5D). In addition, the phosphotyrosine $39 \alpha$-synuclein levels are not significantly different between the different mouse strains in the soluble brain stem fractions (Supplemental Figure 6, A and D), but they are increased in the insoluble brain stem fraction of BCR-ABL hA53T $\alpha$-syn PrP$t T A$ mice (Supplemental Figure 6, B and D). In the $c-A b l^{K O}$ hA53T $\alpha-$ syn mice, phosphotyrosine $39 \alpha$-synuclein levels are unchanged in the soluble fraction (Supplemental Figure 6, E and G) and markedly reduced in the insoluble fraction (Supplemental Figure 6, F and $\mathrm{H}$ ). There is also an increase in phosphotyrosine $39 \alpha$-synuclein and pathologic accumulation of $\alpha$-synuclein and phosphoserine $129 \alpha$-synuclein in the BCR-ABL PrP-tTA mice versus age-matched nontransgenic mice (Supplemental Figure 6, I and J).

Phosphotyrosine $39 \alpha$-synuclein is elevated in sporadic PD and accumulates in Lewy bodies. The potential pathophysiologic relevance of tyrosine 39 phosphorylation of $\alpha$-synuclein was monitored in human postmortem tissues from PD patients and age-matched controls (Supplemental Tables 1 and 2). Total tissue lysates were prepared from substantia nigra, striatum, and cortex. As previously reported, there is a significant increase in tyrosine-phosphorylated c-Abl from PD brain samples over agematched control tissue in substantia nigra and striatum (Figure $8, \mathrm{~A}-\mathrm{D})$. There is also a significant increase in tyrosine 39 phosphorylation of $\alpha$-synuclein in PD substantia nigra and striatum over age-matched controls (Figure 8, A-D). Phosphoserine 129 $\alpha$-synuclein is also increased in the substantia nigra and striatum over age-matched controls (Figure 8, A-D). There is no significant difference between the levels of tyrosine-phosphorylated c-Abl, tyrosine 39 phosphorylation, or serine 129 phosphorylation of $\alpha$-synuclein in the cortex (Figure 8, E and F), a region relatively unaffected in PD (39). Most importantly, there is marked accumulation of phosphotyrosine $39 \alpha$-synuclein in Lewy bodies in PD postmortem brain substantia nigra (Figure $8, \mathrm{G}$ and $\mathrm{H}$ ). In addition, there is also substantial accumulation of phosphoserine 129 $\alpha$-synuclein in Lewy bodies (Figure 8, I and J).

Phosphotyrosine $39 \alpha$-synuclein shows increased propensity for aggregation. Since phosphotyrosine $39 \alpha$-synuclein increases with aging and disease progression in hA53T $\alpha$-syn mice, we asked whether phosphorylation of $\alpha$-synuclein at tyrosine 39 plays any role in aggregation. To determine this, we used a previously described cell-based aggregation assay system (40). Myc-tagged WT $\alpha$-synuclein alone or along with kinase-active c-Abl (c-Abl$\mathrm{KA})$ or the KD version, or myc-tagged WT-Y39F $\alpha$-synuclein along with c-Abl-KA, was overexpressed in HEK293T cells, and immunostaining with antibodies against myc epitope tag on the expressed $\alpha$-synuclein proteins was performed to quantify the aggregates. Increased aggregation of $\alpha$-synuclein is observed only when $\alpha$-synuclein is co-overexpressed with c-Abl-KA, but not with the $\mathrm{KD}$ version of $\mathrm{c}-\mathrm{Abl}$, nor did we observe increased aggregation with co-overexpression of WT-Y39F $\alpha$-synuclein and c-Abl-KA (Figure 9, A and B). To further assess the role of tyrosine 39 phosphorylation in $\alpha$-synuclein aggregation, WT, A53T, A53T-Y39F, or A53T-Y39E $\alpha$-synuclein was overexpressed in HEK293T cells (Figure 9, E and F) or human neurons (Supplemental Figure 7), and aggregation of $\alpha$-synuclein was assessed. A53T $\alpha$-synuclein shows a marked increase in aggregation compared with the WT $\alpha$-synuclein, whereas the A53T phospho-deficient mutant (A53T-Y39F) shows significantly reduced aggregation (Figure 9, E and F, and Supplemental Figure 7, A and B). The phosphomimetic A53T-Y39E $\alpha$-synuclein exhibits aggregation similar to that of A53T $\alpha$-synuclein. Quantification of immunoblots ensures that an equivalent level of myc-tagged $\alpha$-synuclein is expressed (Figure 9, C, D, G, and $\mathrm{H}$ ), indicating that the observed differences in aggregation are not due to differences in $\alpha$-synuclein expression level. To confirm that $\alpha$-synuclein punctates are fibrillar aggregates, thioflavin $S$ and $\alpha$-synuclein dual immunostaining was performed to examine whether $\alpha$-synuclein punctates are thioflavin S positive (Figure 9I). The $\alpha$-synuclein punctates show complete colocalization with thioflavin $S$, indicating that these punctates are fibrillar aggregates of $\alpha$-synuclein. Taken together, our results strongly suggest that tyrosine 39 phosphorylation of $\alpha$-synuclein promotes the aggregation of $\alpha$-synuclein.

\section{Discussion}

The major finding of this study is that c-Abl activation plays a key role in $\alpha$-synuclein neurodegeneration. Activation of c-Abl leads to tyrosine 39 phosphorylation of $\alpha$-synuclein, which strongly correlates with disease progression in hA53T $\alpha$-syn transgenic mice. Overexpression of a constitutively active form of c-Abl, BCR-ABL, substantially and significantly increases the pathology of hA53T $\alpha-$ syn transgenic mice and leads to DA and NE neuron degeneration in aged WT mice. On the other hand, knockout of c-Abl significantly decreases the pathology of hA53T $\alpha$-syn transgenic mice. Importantly, phosphotyrosine $39 \alpha$-synuclein increases over time as hA53T $\alpha$-syn mice become more symptomatic. In addition, this 

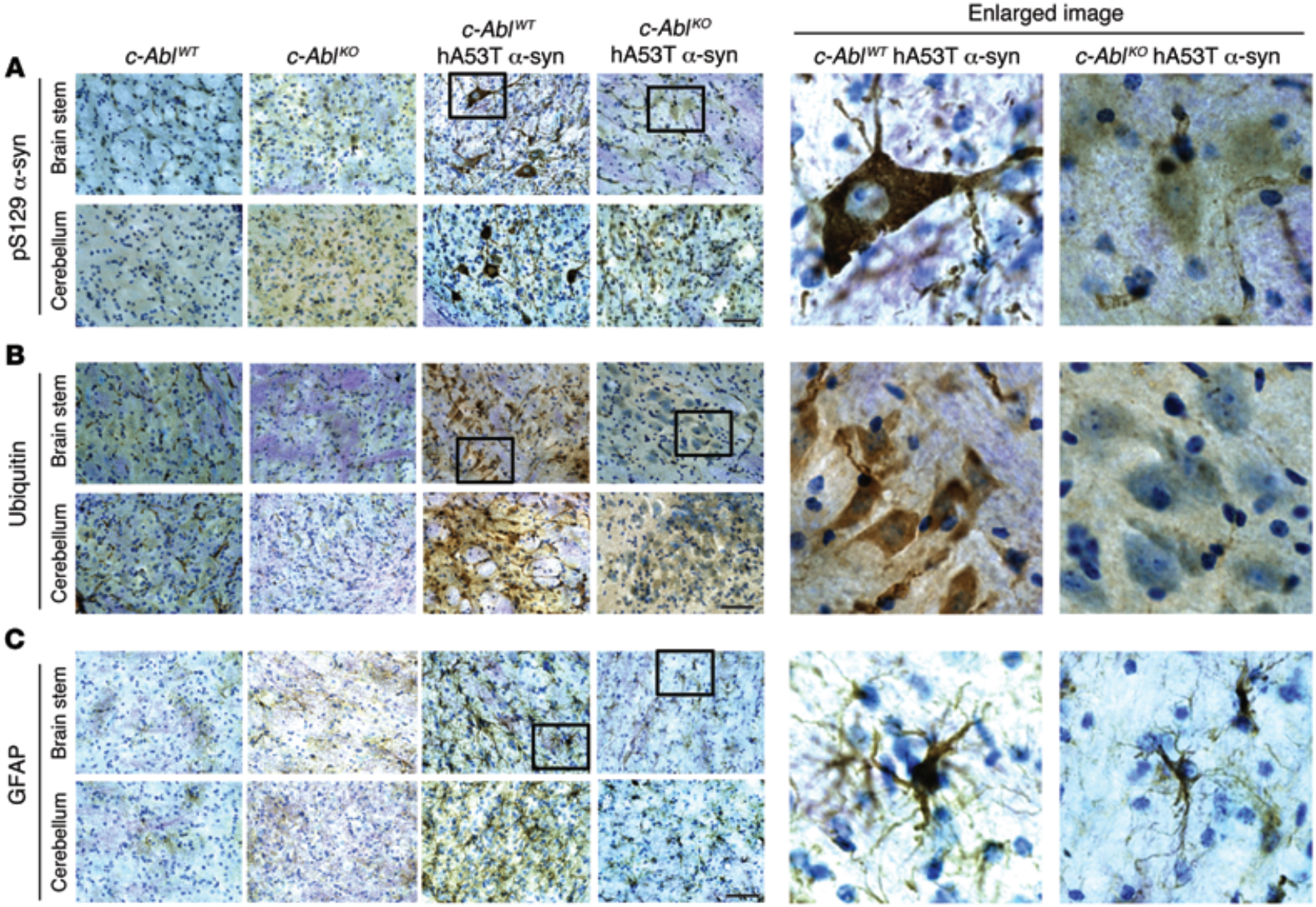

D

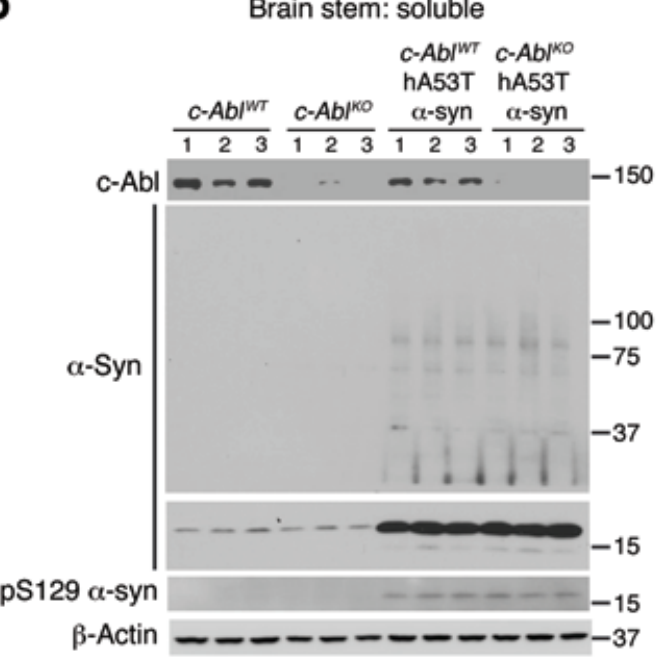

E

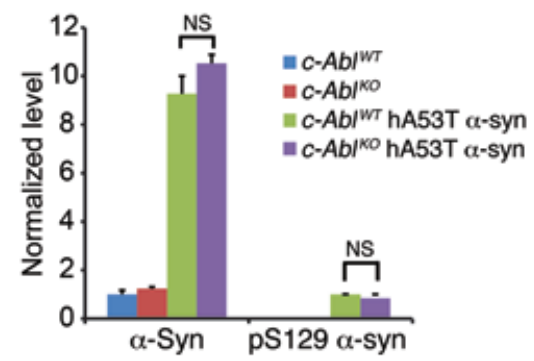

$\mathbf{F}$

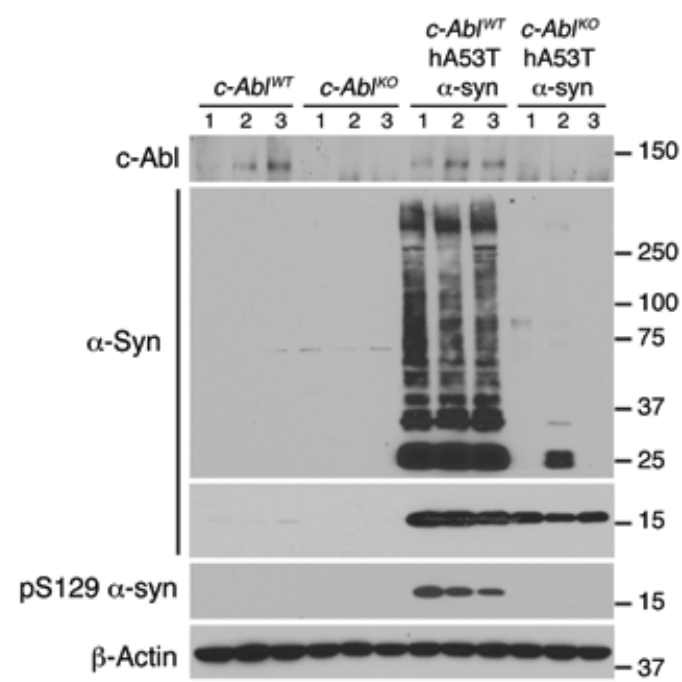

G

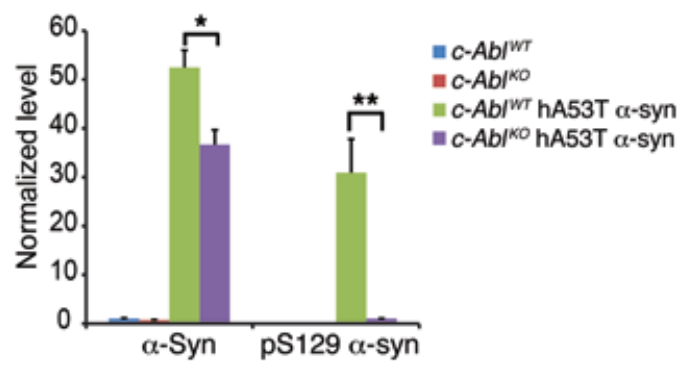


Figure 5. c-Abl deletion reduces $\alpha$-synuclein pathologies. (A-C) Representative pS129 $\alpha$-syn, ubiquitin, and GFAP IHC in the brain stem and cerebellum of 9 -month-old symptomatic $c-A b{ }^{W T}$ hA53T $\alpha$-syn mice versus $c-\left.A b\right|^{K O}$ hA53T $\alpha$-syn mice and age-matched littermate control mice $(n=3$ per group). Enlarged images (zoom-in, $\times 30$; original magnification, $\times 40$ ) of the indicated regions are shown at right. Scale bars: $50 \mu \mathrm{m}$. (D) Representative immunoblots of c-Abl, $\alpha$-syn, pS129 $\alpha$-syn, and $\beta$-actin in the detergent-soluble fraction of brain stem from 9-month-old symptomatic $c$ - $A b^{/ W T}$ hA53T $\alpha$-syn transgenic mice versus $c-\left.A b\right|^{K 0}$ hA53T $\alpha$-syn transgenic mice and age-matched littermate controls. (E) Quantification of $\alpha$-syn monomer and pS129 $\alpha$-syn protein levels in $\mathbf{D}$ normalized to $\beta$-actin and $\alpha$-syn monomer, respectively ( $n=7$ mice per group). (F) Representative immunoblots of c-Abl, $\alpha$-syn, pS129 $\alpha$-syn, and $\beta$-actin in the detergent-insoluble fraction of brain stem from 9-month-old symptomatic $c-A b^{/{ }^{W T}}$ hA53T $\alpha$-syn transgenic mice and age-matched littermate controls. (G) Quantification of $\alpha$-syn monomer and pS129 $\alpha$-syn protein levels in $\mathbf{F}$ normalized to $\beta$-actin and $\alpha$-syn monomer, respectively ( $n=7$ mice per group). (D-C) Data are from 3 independent experiments. Statistical significance was determined by 1-way ANOVA with Tukey's post-test of multiple comparisons. Quantified data are expressed as the mean \pm SEM. ${ }^{*} P<0.05,{ }^{* *} P<0.01$.

is the first report showing that phosphotyrosine $39 \alpha$-synuclein is elevated in the substantia nigra and striatum of PD postmortem brains and that it accumulates in the Lewy bodies. Phosphorylation of $\alpha$-synuclein at tyrosine 39 seems to be a key posttranslational modification driving aggregation. Taken together, our data strongly suggest that c-Abl is an important contributor to the pathogenesis of $\alpha$-synuclein-induced neurodegeneration and that phosphotyrosine $39 \alpha$-synuclein may be a progression marker for $\mathrm{PD}$ and related $\alpha$-synucleinopathies, and that it also plays a central role in $\alpha$-synuclein aggregation.

Phosphorylation of $\alpha$-synuclein has been studied extensively (41), particularly with regard to phosphoserine $129 \alpha$-synuclein $(18,42)$. Phosphorylation of serine 129 of $\alpha$-synuclein is thought to be a marker of $\alpha$-synuclein pathology since $90 \%$ of $\alpha$-synuclein found in Lewy bodies is phosphorylated at serine 129 (42) and the levels increase with the burden of Lewy bodies (43). Controversially, phosphoserine $129 \alpha$-synuclein is thought to contribute to the pathogenesis of PD and related $\alpha$-synucleinopathies (42). We find that the level of phosphoserine $129 \alpha$-synuclein is elevated in the detergent-soluble fraction of hA53T $\alpha$-syn mice as early as 2 months of age. However, the level of phosphoserine $129 \alpha$-synuclein in the detergent-soluble fraction does not change over time, but becomes elevated in the detergent-insoluble fraction when the mice become symptomatic at 7-8 months of age when high-molecular weight species of $\alpha$-synuclein become manifest. Phosphoserine $129 \alpha$-synuclein has been investigated as a potential biomarker in PD, and its concentrations correlated weakly with PD severity (44). In addition, $\alpha$-synuclein levels alone are not sufficient as a PD biomarker (45). Combined total $\alpha$-synuclein and phosphoserine $129 \alpha$-synuclein levels might distinguish different parkinsonian disorders (44). Thus, the utility of total $\alpha$-synuclein and serine 129 phosphorylation of $\alpha$-synuclein does not seem to be overly promising $(44,45)$. In particular, phosphoserine $129 \alpha$-synuclein does seem to be a suitable progression marker in human PD; whether it will be suitable as a diagnostic marker requires further study (44).

On the other hand, detergent-soluble phosphotyrosine 39 $\alpha$-synuclein shows a marked increase in asymptomatic mice from 2 months to 4 and 6 months of age and then decreases, but remains significantly high in symptomatic mice compared with 2-monthold hA53T $\alpha$-syn mice. This decrease after 6 months of age is likely due to its shift to the insoluble fraction. It is also mildly to moderately elevated in the detergent-insoluble fraction in asymptomatic mice, but the levels of detergent-insoluble phosphotyrosine $39 \alpha$-synuclein increase dramatically with disease severity in hA53Ta-syn mice, similarly to the dramatic increase in the level of phosphoserine $129 \alpha$-synuclein in the detergent-insoluble fraction. Since phosphotyrosine $39 \alpha$-synuclein increases throughout the disease course of the hA53T $\alpha$-syn transgenic mice, whereas phosphoserine $129 \alpha$-synuclein levels are stable until $\alpha$-synuclein shifts to the detergent-insoluble fraction, phosphotyrosine $39 \alpha$-synuclein levels may be a more robust disease and progression indicator for PD. Future studies exploring this possibility are warranted.

Recently, Mahul-Mellier and colleagues (46) reported that c-Abl phosphorylated $\alpha$-synuclein on both tyrosine 39 and 125 with only $66 \%$ coverage of the $\alpha$-synuclein protein by mass spectrometry. Since no mass spectrometry data were provided for phosphorylation of tyrosine 125 , and we had $100 \%$ coverage of $\alpha$-synuclein, it is difficult to compare our findings with theirs. In addition, our mutational analysis strongly suggests that tyrosine 39 is exclusively phosphorylated by c-Abl. In contrast to our findings, Mahul-Mellier and colleagues did not observe an increase in tyrosine 39 phosphorylation of $\alpha$-synuclein in PD; however, their analysis was limited to the cortex, which is not affected and where we also did not observe an increase.

Overactivation of c-Abl is emerging as a consistent feature of sporadic PD $(23,25,46-48)$. c-Abl levels as well as markers of c-Abl activity are increased in sporadic PD $(23,25,46-48)$. c-Abl overexpression markedly accelerates the behavioral abnormalities and pathology of hA53T $\alpha$-syn mice and shortens their lifespan. More importantly, c-Abl overexpression in WT mice leads to $\mathrm{DA}$ neuron degeneration at 2 years of age, suggesting that $\mathrm{c}-\mathrm{Abl}$ alone is sufficient to cause neurodegeneration. Accompanying the degeneration of DA neurons is an elevation of phosphotyrosine $39 \alpha$-synuclein and pathologic accumulation of $\alpha$-synuclein, suggesting that phosphotyrosine $39 \alpha$-synuclein may contribute to the degeneration of DA neurons. Other reports further suggest that c-Abl activation is associated with accumulation of $\alpha$-synuclein in the mouse models of $\alpha$-synuclein overexpression $(25,46)$. Interestingly, c-Abl also phosphorylates parkin on tyrosine 143 , leading to its inactivation and accumulation of parkin substrates $(23,24,26)$, raising the possibility that elevation of parkin substrates may contribute to the pathogenesis of sporadic PD (49). c-Abl inhibitors may also affect neuroinflammation induced by $\alpha$-synuclein and facilitate autophagic clearance of $\alpha$-synuclein $(50,51)$. Inhibition of c-Abl may also be protective by inhibiting p38 $\alpha$ (52). In addition, $c-A b l$ inhibition may also have symptomatic effects as it normalizes striatal motor behaviors in the 1-methyl-4phenyl-1,2,3,6-tetrahydropyridine (MPTP) model of PD (53). Future studies are indicated to determine the major pathway by which c-Abl inhibition provides neuroprotection.

Previous studies indicate that brain-penetrant c-Abl inhibitors protect against MPTP neurotoxicity $(26,48)$ and normalize striatal motor behaviors (53), as well as reverse the loss of DA neurons and improve motor behavior in a viral $\alpha$-synuclein model of PD (25). However, a major caveat of these reports is that the inhibitors 
A

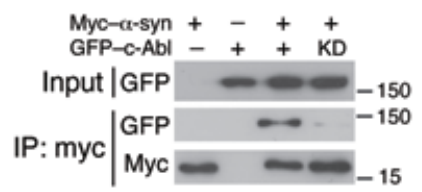

B

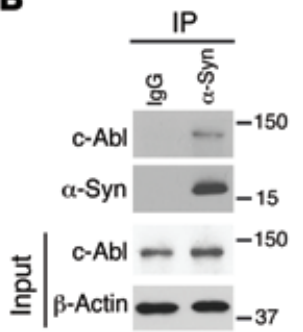

C

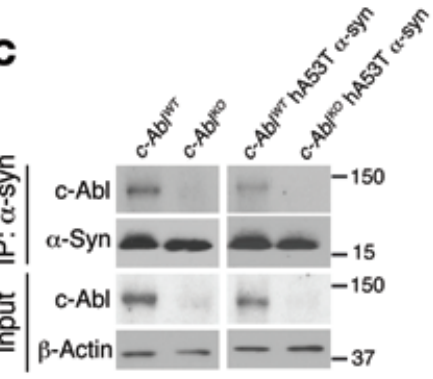

D

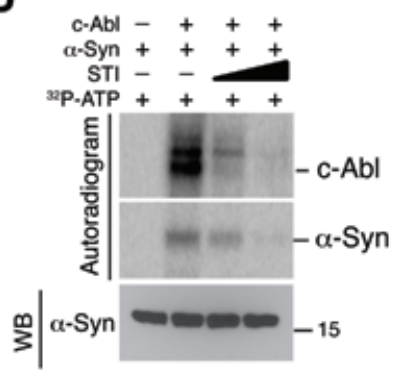

$\mathbf{E}$

MDVFMKGLSK AKEGVVAAAE KTKQGVAEAA GKTKEGVLYV GSKTKEGVVH GVATVAEKTK EQVTNVGGAV VTGVTAVAQK TVEGAGSIAA ATGFVKKDQL GKNEEGAPQE GILEDMPVDP DNEAYEMPSE EGYQDYEPEA

$100 \%$ sequence coverage
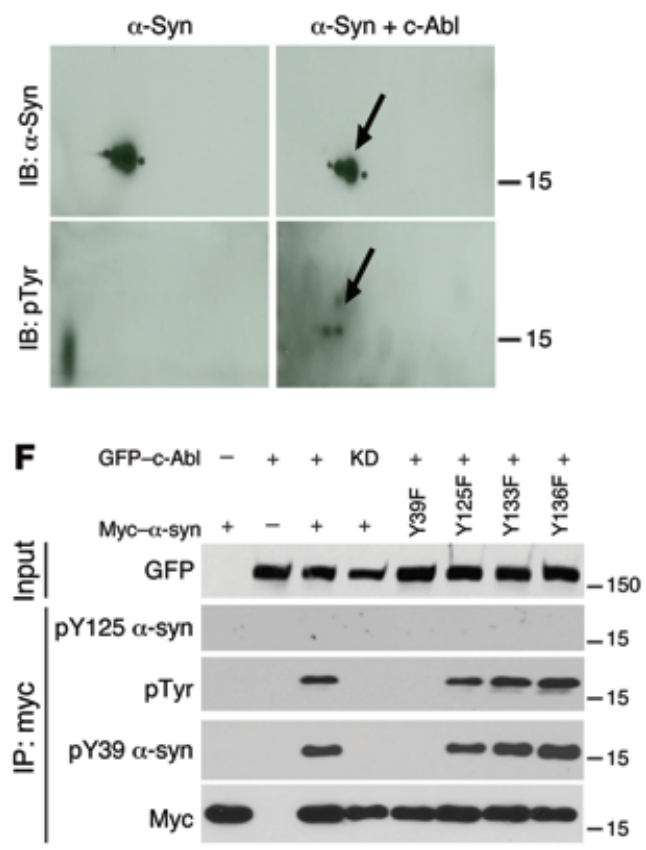

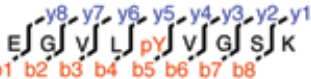

b1 $1^{\circ} 2^{\circ} 3^{\circ}$ b4 $4^{\circ}$ b5 $5^{\circ} 6^{\circ} 7^{\circ}$ b8 pY39

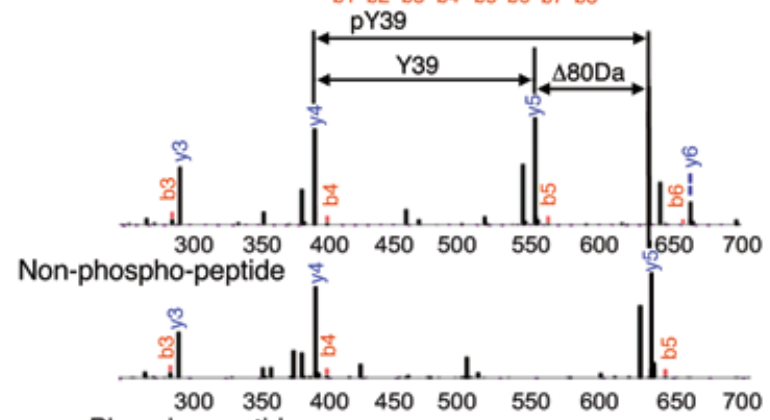

Phospho-peptide

Figure 6. c-Abl interacts with and phosphorylates $\alpha$-synuclein. (A) Coimmunoprecipitation of myc-tagged $\alpha$-syn (myc- $\alpha$-syn) and GFP-tagged c-Abl (GFPc-Abl) by anti-myc antibody in SH-SY5Y cells cotransfected with myc- $\alpha$-syn and GFP-c-Abl or kinase-dead (KD) (lysine 290 arginine) version of c-Abl (GFPc-Abl-KD) followed by IB. (B) Coimmunoprecipitation of $\alpha$-syn and c-Abl by anti- $\alpha$-syn antibody in the brain stem from nontransgenic mice followed by IB. Anti-IgG was used as a negative control. (C) Coimmunoprecipitation of $\alpha$-syn and c-Abl by anti- $\alpha$-syn antibody in the brain tissue lysates from WT, c-Abl knockout $\left(c-A b^{K 0}\right)$, hA53T $\alpha$-syn, and $c$-Ab/K0 hA53T $\alpha$-syn mice followed by IB. (D) In vitro kinase assay showing that c-Abl phosphorylates $\alpha$-syn. Autoradiogram indicates that STI-571, a c-Abl kinase inhibitor, dose-dependently reduced phosphorylation of $\alpha$-syn and c-Abl. Immunoblot in the bottom panel shows equivalent amount of $\alpha$-syn used in the experiment. (E) c-Abl phosphorylates $\alpha$-syn on tyrosine ( $Y$ ) 39. Mass spectrometric analysis reveals $100 \%$ sequence coverage of $\alpha$-syn, showing that all tyrosine residues were investigated for phosphorylation status. Phosphorylated $Y 39$ is indicated in red; other tyrosines are indicated in green (top). $\alpha$-Syn phosphorylated by c-Abl was separated by 2-DE followed by IB (bottom left). The arrows indicate tyrosine phosphorylation of $\alpha$-syn. Both nonphosphorylated and phosphorylated $\alpha$-syn were subjected to liquid chromatography-tandem mass spectrometry (LC-MS/MS) to identify the phosphorylation site (bottom right). LC-MS/MS spectra of the nonphosphorylated peptide (ECVLYVCSK) and the phosphorylated peptide (EGVLpYVCSK) are compared, demonstrating that there is the 80-Da shift for the Y39 ion containing the phosphate moiety. The phosphorylated amino acid is preceded by a "p" and highlighted in red. (F) IP of myc- $\alpha$-syn by anti-myc antibody in SH-SY5Y cells cotransfected with indicated plasmids followed by IB with anti-myc, antipY39 $\alpha$-syn, anti-pTyr, or anti-pY125 $\alpha$-syn antibodies. Inputs were immunoblotted with anti-GFP antibodies. All experiments were repeated at least 3 times.

used in these studies have a broad range of inhibitory activities on several kinases. They are nonspecific kinase inhibitors, and thus one cannot conclude that c-Abl inhibition accounts for the neuroprotection observed. For instance, nilotinib, which was shown to be protective in a viral $\alpha$-synuclein model (25), has several kinase targets (54). Nilotinib inhibits the following kinases (listed in the order of inhibitory potencies): DDR1 $>$ DDR2 $>$ BCR-ABL $>$ PDG$\mathrm{FR} \alpha / \beta>\mathrm{KIT}>\mathrm{CSF}-1 \mathrm{R}$. In addition nilotinib has also been found to affect MAPK activity (55). Therefore, due to this well-known nonselectivity of nilotinib, one cannot make a definitive conclusion regarding the role of $\mathrm{c}-\mathrm{Abl}$ activation in the pathogenesis of PD and $\alpha$-synucleinopathies. Using both BCR-ABL overexpression 
A

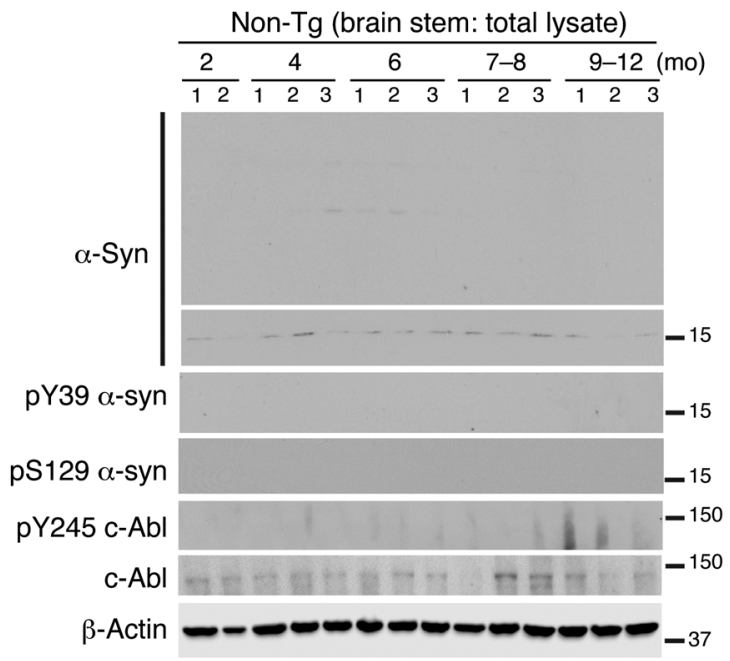

B

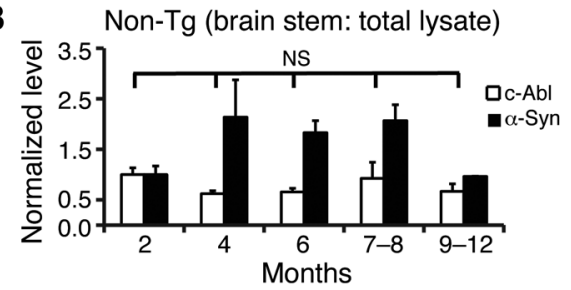

E hA53T $\alpha$-syn (brain stem: insoluble)

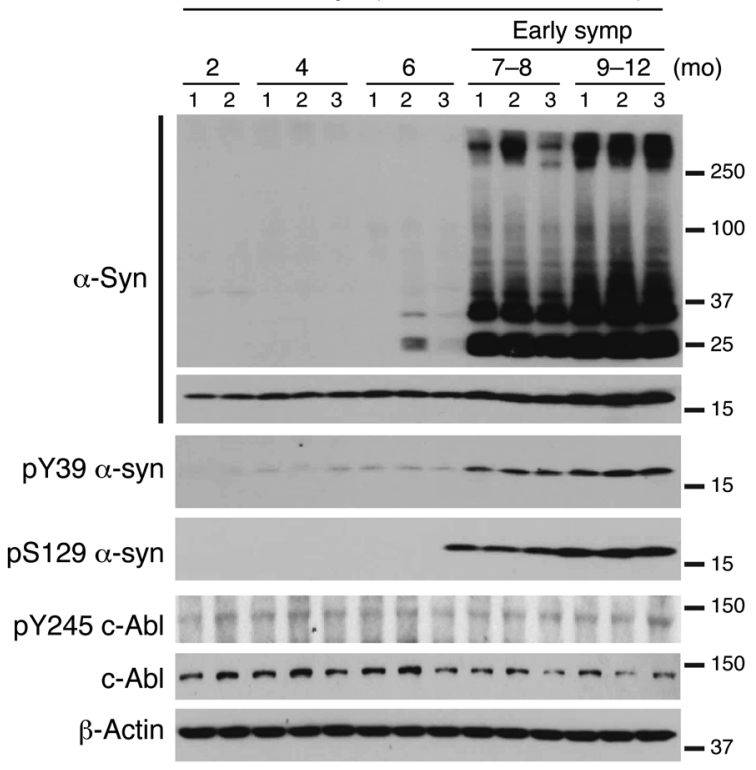

C
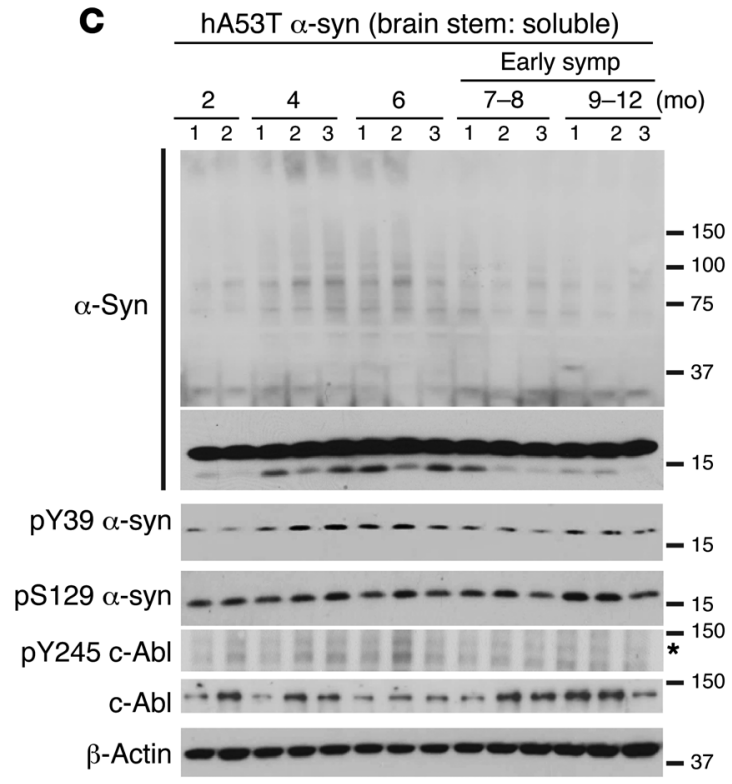

D hA53T $\alpha$-syn (brain stem: soluble)

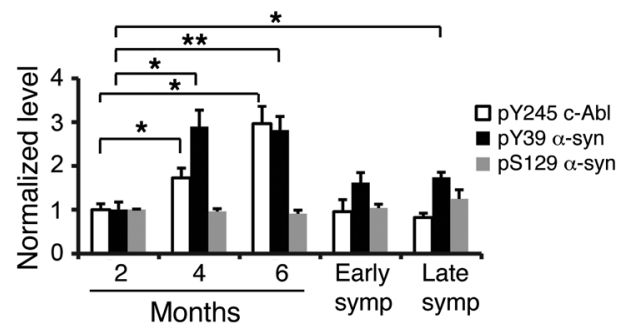

F hA53T $\alpha$-syn (brain stem: insoluble)

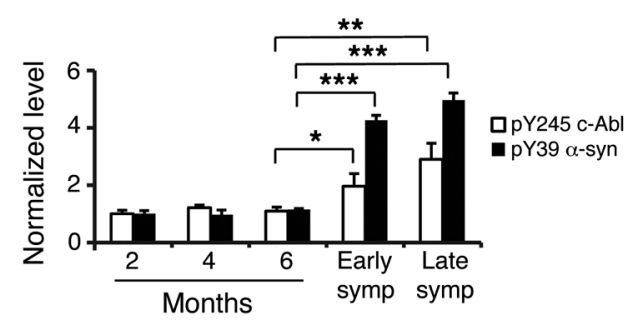

G hA53T $\alpha$-syn (brain stem: insoluble)

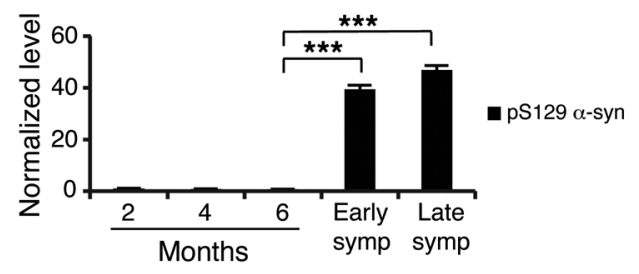

Figure 7. Phosphotyrosine $39 \alpha$-synuclein increases in the brain stem of hA53T $\alpha$-syn transgenic mice. (A) Representative immunoblots of $\alpha$-syn, pY39 $\alpha$-syn, pS129 $\alpha$-syn, pY245-c-Abl, c-Abl, and $\beta$-actin in the brain stem from nontransgenic mice of different ages. (B) Quantification of $\alpha$-syn monomer and c-Abl protein levels in A normalized to $\beta$-actin ( $n=5$ mice per group). (C) Representative immunoblots of $\alpha$-syn, pY39 $\alpha$-syn, pS129 $\alpha$-syn, pY245-c-Abl, c-Abl, and $\beta$-actin in the detergent-soluble fraction of brain stem from hA53T $\alpha$-syn transgenic mice of different ages. Asterisk indicates nonspecific band. (D) Quantification of pY245-c-Abl protein level normalized to c-Abl and pY39 $\alpha$-syn and pS129 $\alpha$-syn protein levels normalized to $\alpha$-syn monomer in $\mathbf{A}$ ( $n=5-10$ mice per group). (E) Representative immunoblots of $\alpha$-syn, pY39 $\alpha$-syn, pS129 $\alpha$-syn, pY245-c-Abl, c-Abl, and $\beta$-actin in the detergent-insoluble fraction of brain stem from hA53T $\alpha$-syn transgenic mice of different ages. (F and $\mathbf{G}$ ) Quantification of pY245-c-Abl protein level normalized to c-Abl and pY39 $\alpha$-syn and pS129 $\alpha$-syn protein levels normalized to $\alpha$-syn monomer in $\mathbf{E}$ ( $n=5-10$ mice per group). Data are from 3 independent experiments. Statistical significance was determined by 1-way ANOVA with Tukey's post-test of multiple comparisons. Quantified data are expressed as the mean \pm SEM. ${ }^{*} P<0.05,{ }^{* *} P<0.01,{ }^{* * *} P<0.001$. 
A

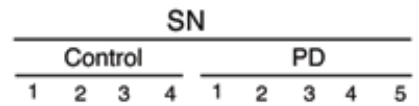

c-Abl

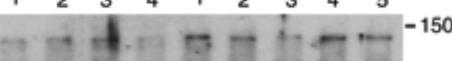

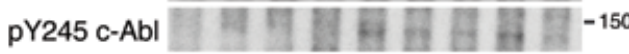

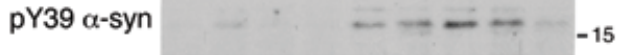

pS129 $\alpha$-syn

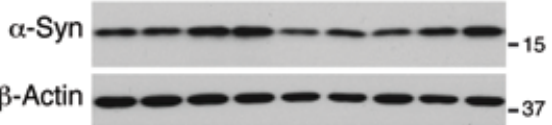

B

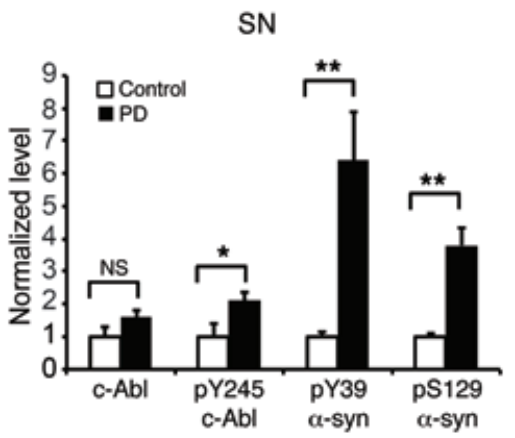

E
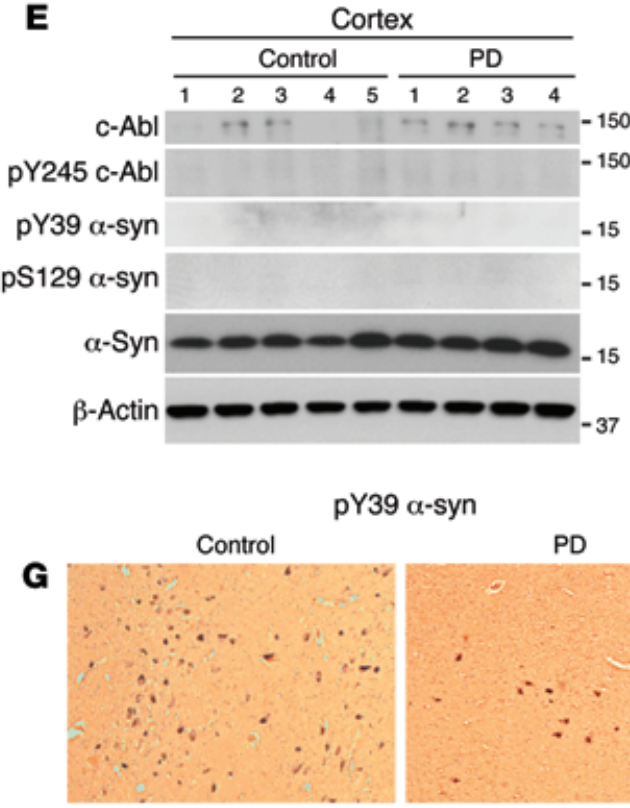

pY39 $\alpha$-syn

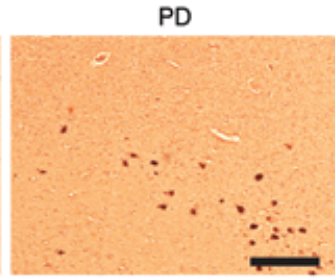

H

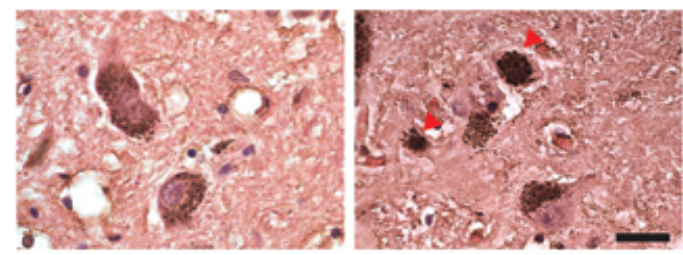

C

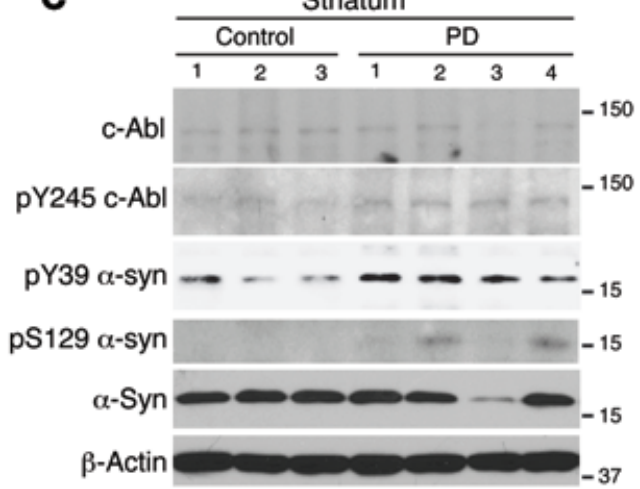

D

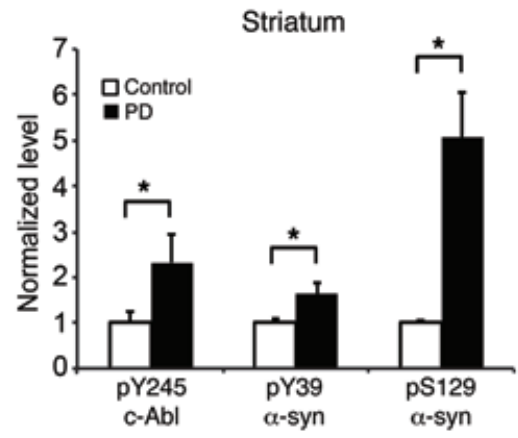

$\mathbf{F}$

Cortex
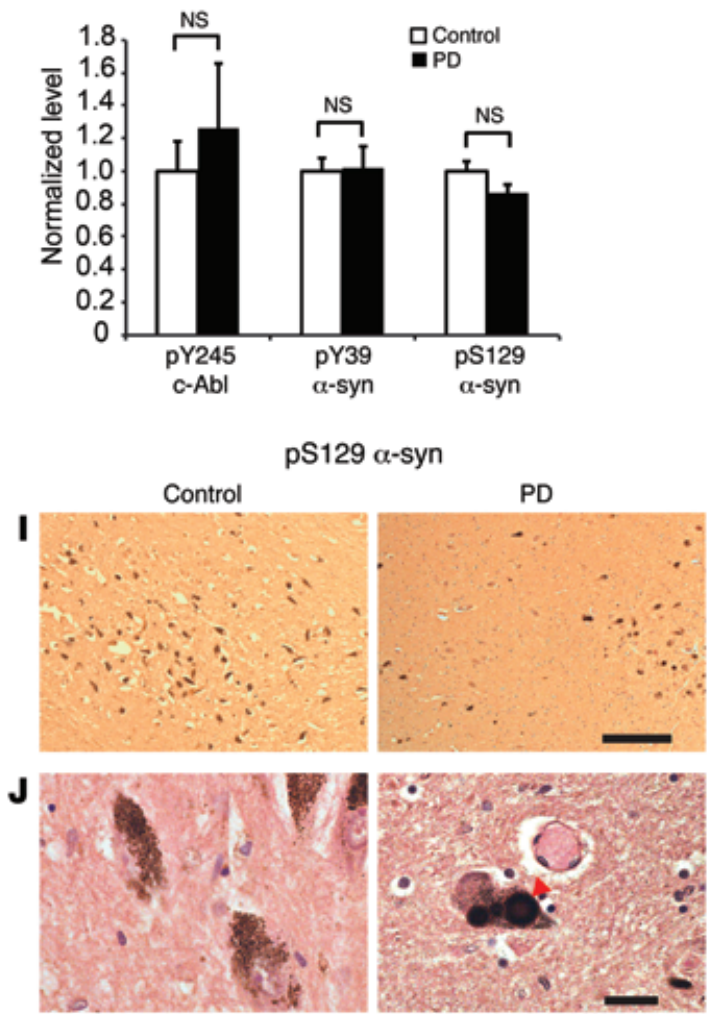

Figure 8. Phosphotyrosine $39 \alpha$-synuclein level is elevated in PD patients and accumulates in Lewy bodies. (A, C, and E) Representative immunoblots of c-Abl, pY245 c-Abl, pY39 $\alpha$-syn, pS129 $\alpha$-syn, $\alpha$-syn, and $\beta$-actin in the total lysates from substantia nigra, striatum, and cortex regions from PD patient brains and age-matched controls. (B) Quantification of c-Abl protein level normalized to $\beta$-actin, pY245 c-Abl protein level normalized to c-Abl, and pY39 $\alpha$-syn and pS129 $\alpha$-syn protein levels normalized to $\alpha$-syn in the substantia nigra (control $n=5, P D n=8$ ). (D and F) Quantification of pY245 c-Abl protein level normalized to c-Abl and pY39 $\alpha$-syn and pS129 $\alpha$-syn protein levels normalized to $\alpha$-syn in the striatum (control $n=5, P D n=7$ ) (D) and cortex (control $n=7, P D n=6$ ). Data are from 3 independent experiments. Statistical significance was determined by 2-tailed unpaired Student's $t$ test. Quantified data are expressed as the mean $\pm \mathrm{SEM}$. ${ }^{*} P<0.05$, ${ }^{*} P<0.01$. (G and $\left.\mathbf{I}\right)$ Representative $\mathrm{pY} 39 \alpha$-syn and $\mathrm{pS} 129 \alpha$-syn IHC in the substantia nigra from PD patient brains and age-matched controls $(n=2)$. Scale bars: $250 \mu \mathrm{m}$. (H and J) High-magnification images of $\mathbf{G}$ and $\mathbf{~ I}$. Scale bars: $25 \mu \mathrm{m}$. 
and conditional $c$ - $A b l$ knockouts, we provide, to our knowledge, the first conclusive evidence that c-Abl activation plays a role in the pathogenesis of $\alpha$-synucleinopathies and PD, strongly implicating that inhibition of c-Abl alone could be neuroprotective. Since the c-Abl inhibitors currently in use have substantial toxicity and side effects due to their broad range of activities, our study provides a strong rationale to develop selective, brain-penetrant c-Abl inhibitors with better safety records than the current c-Abl inhibitors used in oncology clinics. If future studies indicate that markers of c-Abl activation such as phosphotyrosine 245 of c-Abl, phosphotyrosine 143 of parkin, and/or phosphotyrosine 39 of $\alpha$-synuclein are detectable in the cerebrospinal fluid of PD patients, inhibition of c-Abl in the treatment of PD may be particularly attractive, since one would then have a tool to monitor treatment efficacy.

The strong correlation between phosphotyrosine $39 \alpha$-synuclein levels and the development of neuropathology as observed in our mouse models suggests that phosphotyrosine $39 \alpha$-synuclein may play a role in neurodegeneration. Consistent with this notion, phosphotyrosine $39 \alpha$-synuclein is found in Lewy bodies of PD brain. Our cell culture-based aggregation assays using HEK293T cells indicate that c-Abl increases $\alpha$-synuclein aggregation, which is significantly prevented by the KD version of c-Abl or by the phospho-deficient mutant of $\alpha$-synuclein (Y39F). In addition, aggregation assays using HEK293T cells as well as human neuronal culture further indicate that phospho-deficient A53T $\alpha$-synuclein (A53TY39F $\alpha$-synuclein), but not the phosphomimetic mutant (A53TY39E $\alpha$-synuclein), markedly reduces aggregation, suggesting that phosphotyrosine $39 \alpha$-synuclein plays a role in aggregation. Since aggregation of $\alpha$-synuclein is thought to play a central role in its neurotoxicity, our results strongly imply that phosphotyrosine 39 $\alpha$-synuclein may play a role in neurodegeneration. Future studies will be required to directly test this possibility.

In summary, we report a key and defining role for c-Abl activation in $\alpha$-synuclein-induced neurodegeneration. In particular, our study implicates that selective inhibition of c-Abl could be neuroprotective. In addition, the strong correlation of phosphotyrosine $39 \alpha$-synuclein with disease progression in hA53T $\alpha$-syn mice, as well as its increased propensity for aggregation, suggests that phosphotyrosine $39 \alpha$-synuclein could be a progression marker and contribute to the pathogenesis of PD and $\alpha$-synucleinopathies.

\section{Methods}

Human postmortem brain tissues for IB analysis. Substantia nigra, striatum, and cortex samples from neurologically unimpaired subjects ( $n=5$ for each region) and from subjects with PD ( $n=8$ for substantia nigra, $n=7$ for striatum and for cortex) were obtained from the Division of Neuropathology, Department of Pathology, Johns Hopkins University. Diagnosis of PD was confirmed by pathologic and clinical criteria. The average age of subjects was $78.2 \pm 1.6$ years in the PD group and $76.3 \pm 3.6$ years in the control group $(P=0.62)$. Average postmortem delay was 12.45 days and was not significantly different between the 2 groups $(P=0.36)$.

Animals. The generation of transgenic mice that overexpress human A53T $\alpha$-synuclein using the mouse prion protein $(\operatorname{PrP})$ promoter has been described previously (27). Tetracycline-controllable $B C R-A B L$ transgenic mice [FVB/N-Tg(tetO-BCR/ABL1)2Dgt/J] were purchased from The Jackson Laboratory. Mice possessing the lox-
P-flanked $c-A b l$ gene exon $5\left(c-A b l^{\left.f t / f-N e s t i n-C r e^{+}\right)}\right.$were previously described and were used in this study (37). Randomized mixed-gender cohorts were used for all animal experiments. All mice were acclimatized for 3 days in the procedure room before any experiments were started. We have taken great effort to reduce animal suffering from pain and discomfort.

Antibodies. Rabbit polyclonal phosphotyrosine $39 \alpha$-synuclein antibodies were generated as described below. Primary antibodies used include the following: mouse anti- $\alpha$-synuclein (610787; BD Transduction Laboratories), mouse anti- $\alpha$-synuclein LB509 (ab27766; Abcam), mouse anti- $\alpha$-synuclein Syn 204 (Santa Cruz Biotechnology), rabbit anti- $\alpha$-synuclein (2642; Cell Signaling Technology), rabbit anti-pY245 c-Abl (2861; Cell Signaling Technology), mouse anti-c-Abl (554148; BD Transduction Laboratories), mouse anti-pS129 $\alpha$-synuclein (pSyn\#64) (015-25191; Wako), rabbit monoclonal anti-pS129 $\alpha$-synuclein (ab168381; Abcam), rabbit anti-BCR-ABL (3902; Cell Signaling Technology), mouse anti-ubiquitin (MAB1510; Millipore), rabbit antiGFAP (Z0334; Dako), rabbit anti-Y125 $\alpha$-synuclein (ab124955; Abcam), rabbit anti-phosphotyrosine (610009; BD Transduction Laboratories), rabbit anti-GFP (ab290; Abcam), and mouse anti-c-myc (Roche Diagnostics). Secondary antibodies used include the following: phosphotyrosine, 4G10 platinum-peroxidase (Millipore), actin-peroxidase (Sigma-Aldrich), myc-peroxidase (Roche Diagnostics), anti-rabbit and anti-mouse IgG (Santa Cruz Biotechnology), anti-phosphotyrosine, and peroxidase-linked species-specific whole antibody (GE Healthcare).

Plasmid constructions. The full-length WT human $\alpha$-synuclein was cloned into pRK5-myc vector (Stratagene). The full-length WT and A53T human $\alpha$-synucleins cloned into pCMV5 used for expressions in HEK293T cells or human neurons were gifts from Thomas C. Sudhof (Department of Molecular and Cellular Physiology, Howard Hughes Medical Institute, Stanford University School of Medicine, Stanford, CA). To generate human $\alpha$-synuclein mutant plasmids (WT-Y39F, WT-Y125F, WT-Y133F, WT-Y136F, A53T-Y39F, and A53T-Y39E), site-directed mutagenesis was carried out using the QuikChange site-directed mutagenesis kit (Stratagene) with the pRK5-myc-WT $\alpha$-synuclein or pCMV5-myc-WT $\alpha$-synuclein plasmids as templates. All mutation sites were confirmed by DNA sequencing analysis. GFPc-Abl-KA (kinase-active) and GFP-c-Abl-KD (kinase-dead) were provided by Z.M. Yuan (Department of Genetics and Complex Diseases, Harvard T.H. Chan School of Public Health, Boston, MA).

Reagents. The following chemicals were used: c-Abl recombinant protein (SE 290; BIOMOL International), $\alpha$-synuclein human recombinant protein (55456; Anaspec), c-Abl kinase inhibitor STI571 (Novartis Pharma AG), Phosphatase Inhibitor Cocktail II and III [P5726 (II), P044 (III)], thioflavin S (Sigma-Aldrich), citrate-based antigen unmasking solutions (Vector Laboratories), and Lipofectamine Plus reagent and Lipofectamine 2000 (Invitrogen).

Immunofluorescence analysis. Immunofluorescence in tissue sections was performed as described previously (56) with some modifications. $4 \%$ paraformaldehyde/PBS (pH 7.4)-fixed coronal brain sections were blocked with $10 \%$ donkey serum (Sigma-Aldrich)/PBS plus $0.3 \%$ Triton X-100 and incubated with indicated antibodies. After brief washes with PBS, floating brain sections were incubated with corresponding secondary antibodies conjugated with fluorescent dyes (Alexa Fluor 555-conjugated donkey antibody to mouse IgG and Alexa Fluor 488-conjugated donkey antibody to rabbit IgG). Thioflavin S staining was performed as described previously (57). Transfected cells 
A
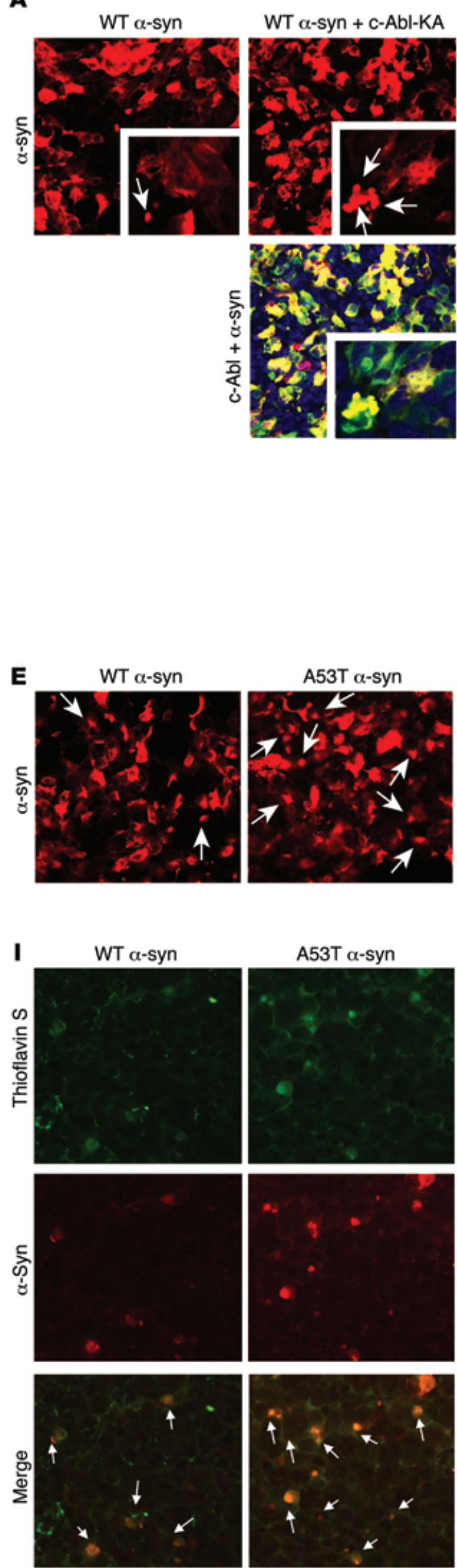
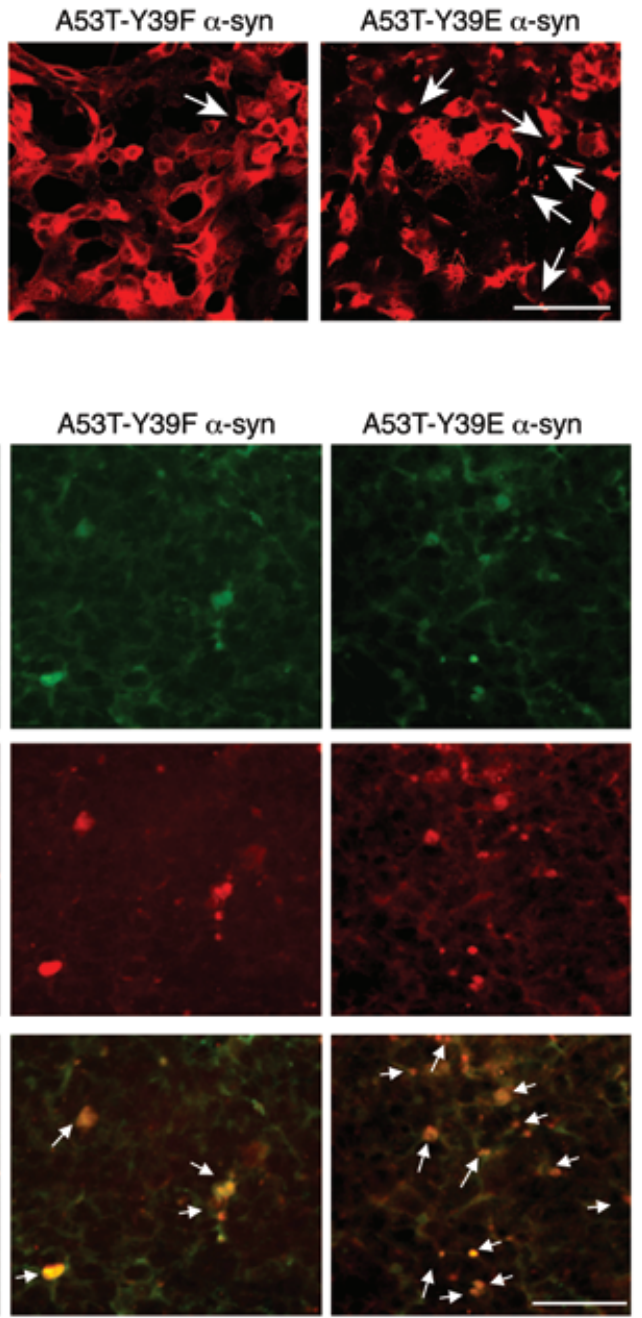
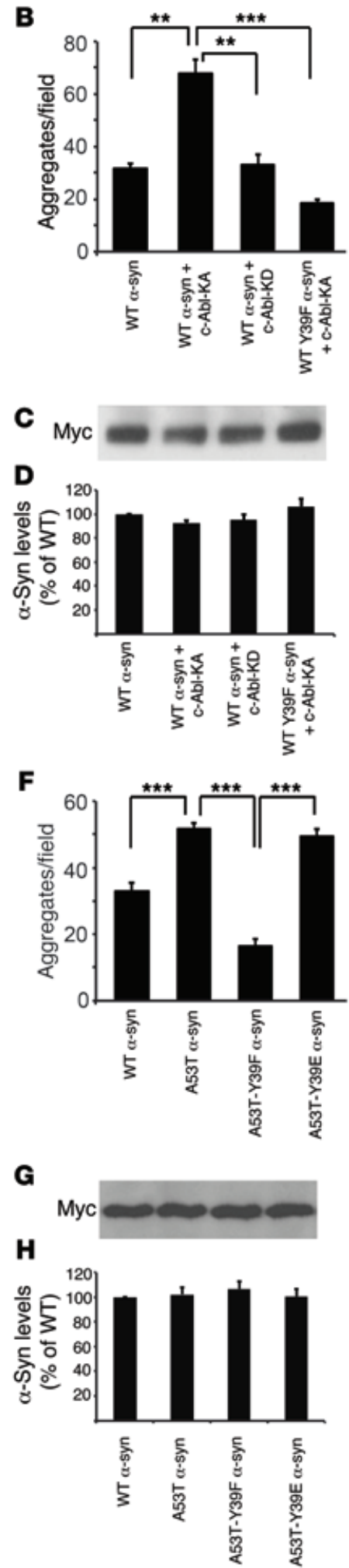
Figure 9. Phosphorylation of $\alpha$-synuclein at tyrosine 39 triggers aggregation. (A and E) Representative immunofluorescent images of myc-tagged $\alpha$-syn (red) or GFP-tagged c-Abl and myc-tagged $\alpha$-syn (merge) in HEK293T cells transfected with indicated plasmids. Single-headed arrows indicate aggregates. Double-headed arrow indicates area with no aggregation. Scale bars: $50 \mu \mathrm{m}$; $5 \mu \mathrm{m}$ (inset). (B and F) Number of immunopositive aggregates per field was quantitated and compared with WT levels. (C and G) Immunoblots of cell lysates with antibodies against the myc epitope. (D and H) Quantifications of myc as percentage of WT levels. (I) Representative immunofluorescent images of thioflavin S staining in HEK293T cells overexpressing indicated myc-tagged $\alpha$-syn. Single-headed arrows indicate colocalization of thioflavin $\mathrm{S}$ and $\alpha$-syn. Scale bar: $25 \mu \mathrm{m}$. Data are from at least 3 independent experiments. (B, D, F, and $\mathbf{H}$ ) Statistical significance was determined by 1-way ANOVA with Tukey's post-test of multiple comparisons. Quantified data are expressed as the mean \pm SEM. ${ }^{* *} P<0.01,{ }^{* *} P<0.001$

were washed with PBS and incubated with $0.01 \%$ thioflavin S (SigmaAldrich) for 8 minutes. Cells were then fixed with $4 \%$ paraformaldehyde for 30 minutes for subsequent immunocytochemistry. Images were obtained using a fluorescent microscope (Zeiss Axiovert 200M) or a confocal microscope (Zeiss Confocal LSM 710). Immunofluorescence in transfected HEK293T cells or human neurons was performed as described previously (40). Transfected HEK293T cells or human neurons were washed 3 times with PBS, and fixed for 20 minutes at room temperature in PBS containing 4\% paraformaldehyde. Following 3 washes with PBS, the fixed cultures were permeabilized for 5 minutes (PBS containing 0.1\% Triton X-100; Sigma-Aldrich). Cells were washed 3 times with PBS and blocked for 20 minutes with 5\% BSA in PBS. $\alpha$-Synuclein expression was monitored using myc antibodies (monoclonal, 1:200; Roche Diagnostics). Images were obtained using a confocal microscope (Zeiss Confocal LSM 710).

Quantitative analysis of aggregates. Immunopositive aggregates were quantified using Image J software. Immunofluorescence images of $\alpha$-synuclein were converted to grayscale, a threshold was set up for the immunostaining based on particle size, and the area of puncta was defined. Based on this parameter, the number of aggregates per field was counted.

IHC for mouse brain. IHC was performed as described previously $(26,56)$. Mice were intracardially perfused with ice-cold PBS and $4 \%$ paraformaldehyde/PBS (wt/vol, pH 7.4) after deep anesthesia by i.p. injection of Nembutal sodium solution $(50 \mu$ l of twofold dilution in PBS of pentobarbital sodium $50 \mathrm{mg} / \mathrm{ml}$; Lundbeck). Brains were removed and postfixed for 16 hours in the same fixative. After cryoprotection in $30 \%$ sucrose/PBS (wt/vol, $\mathrm{pH} 7.4$ ), brains were frozen on dry ice, and serial coronal sections $(40-\mu \mathrm{m}$ sections of brain stem, cerebellum, cortex, hippocampus, and striatum or $60-\mu \mathrm{m}$ sections of midbrain) were cut with a microtome. Free-floating sections were blocked with $4 \%$ goat serum (vol/vol; Sigma-Aldrich)/ PBS plus $0.2 \%$ Triton $\mathrm{X}-100$ (vol/vol) and incubated with indicated antibodies, followed by incubation with biotin-conjugated secondary antibodies to rabbit or mouse, $\mathrm{ABC}$ reagents (Vector Laboratories), and Sigmafast DAB tablets (Sigma-Aldrich). Sections were counterstained with Nissl (0.09\% thionin, wt/vol) after desired antigen staining as described previously (58). A set of cortical, hippocampal, and cerebellar sections were directly stained with Nissl as described above. Sections were dehydrated in $100 \%$ ethanol and cleared in Xylene (Fisher Scientific) followed by mounting with DPX
(Sigma-Aldrich) before imaging under a microscope (Axiophot photomicroscope; Carl Zeiss Vision).

Quantitative analysis of IHC. Every fourth serial section $(60 \mu \mathrm{M}$ for substantia nigra region and $40 \mu \mathrm{M}$ for cortex, hippocampus, cerebellum, brain stem, and striatum regions) was stained for TH and/or Nissl. TH-positive and Nissl-positive neurons from the SNPc and LC regions or only Nissl-positive neurons from cortical, hippocampal, and cerebellar regions and the nucleus basalis were counted through optical fractionators, the unbiased method for cell counting. This method was carried out using a computer-assisted image analysis system consisting of an Axiophot photomicroscope (Carl Zeiss Vision) equipped with a computer-controlled motorized stage (Ludl Electronics), a Hitachi HVC2O camera, and Stereo Investigator software (MicroBrightField). The total number of TH-stained neurons and/or Nissl counts was calculated under $\times 100$ (numerical aperture 1.4, coefficient of error 0.17) magnification (58-61). Serial striatal sections were processed for TH staining following the same procedure as above. Fiber density in the striatum was quantified by optical density (OD). ImageJ software (NIH) was used to analyze the OD as previously described (62).

IHC for PD postmortem brain. Slides with $10-\mu \mathrm{m}$ thickness of formalin-fixed paraffin-embedded human postmortem substantia nigra tissues ( $n=2$ for each of control and PD) were obtained from the Division of Neuropathology, Department of Pathology, Johns Hopkins University (Supplemental Table 2). The tissue sections were deparaffinized and rehydrated, and then heat-induced epitope retrieval was performed with citrate-based antigen unmasking solutions (Vector Laboratories). Then, the slides were stained with rabbit monoclonal phosphoserine $129 \alpha$-synuclein antibodies (ab168381; Abcam) or rabbit polyclonal phosphotyrosine $39 \alpha$-synuclein antibodies. All sections were stained with $\mathrm{H} \& \mathrm{E}$.

Cell culture and transfection. Human neuroblastoma SH-SY5Y cells (ATCC) or HEK293T cells (Invitrogen) were grown in DMEM containing $10 \% \mathrm{FBS}$ and antibiotics in a humidified 5\% CO2/95\% air atmosphere at $37^{\circ} \mathrm{C}$. Human embryonic stem cells (H1; WiCell) were cultured using standard protocol on feeders. For neural induction, cells were harvested with collagenase $(1 \mathrm{mg} / \mathrm{ml})$, and single-cell suspensions were made using Accutase (Sigma-Aldrich). Cells were seeded on Matrigel-coated plates at a density of 40,000 cells $/ \mathrm{cm}^{2}$ in neural induction medium (Stem Cell Technologies) for the first 10 days and then in neurobasal medium supplemented with B27 for the next 21 days. For transient transfection, cells were transfected with $4 \mu$ g (for IP in 100-mm dishes) or $0.5 \mu \mathrm{g}$ (for immunofluorescence in 24-well plates) of each plasmid of target vector using Lipofectamine Plus or Lipofectamine 2000 reagent, according to the manufacturer's instructions (Invitrogen). Two days after transfection, cells were either harvested for IP/IB or fixed in $4 \%$ paraformaldehyde followed by immunostaining.

Tissue lysate preparation. Tissue lysates were prepared as described previously (27) with some modifications. Nonionic detergent-soluble and -insoluble fractions were made by homogenization of tissue in brain lysis buffer (10 mM Tris- $\mathrm{HCl}, \mathrm{pH} 7.4,150 \mathrm{mM} \mathrm{NaCl}, 5 \mathrm{mM}$ EDTA, 0.5\% Nonidet P-40, Phosphatase Inhibitor Cocktail II and III [Sigma-Aldrich], and complete protease inhibitor mixture). The homogenate was centrifuged for 20 minutes at $4^{\circ} \mathrm{C}, 100,000 \mathrm{~g}$, and the resulting pellet (P1) and supernatant (S1, soluble) fractions were collected. The P1 was washed once in brain lysis buffer containing nonionic detergent $(0.5 \%$ Nonidet $\mathrm{P}-40)$, and the resulting pellet (P2, nonionic detergent-insoluble) was homogenized in brain lysis buffer 
containing $1 \%$ SDS and $0.5 \%$ sodium deoxycholate. The homogenate was centrifuged, and the resulting supernatant (nonionic detergent-insoluble) was collected. Total lysates were prepared by homogenization of tissue in RIPA buffer (50 mM Tris, pH 8.0, $150 \mathrm{mM} \mathrm{NaCl}$, $1 \%$ Nonidet P-40, 1\% SDS, 0.5\% sodium deoxycholate, Phosphatase Inhibitor Cocktail II and III [Sigma-Aldrich], and complete protease inhibitor mixture). The homogenate was centrifuged for 20 minutes at $4^{\circ} \mathrm{C}, 100,000 \mathrm{~g}$, and the resulting supernatant was collected.

$I P$ and IB analysis. For coimmunoprecipitation from cell cultures, SH-SY5Y cells were transiently transfected with indicated plasmids. After 48 hours, cells were washed with cold PBS and harvested in IP buffer (1\% Triton X-100, Phosphatase Inhibitor Cocktail II and III [Sigma-Aldrich], and complete protease inhibitor mixture in PBS). The lysate was then rotated at $4^{\circ} \mathrm{C}$ for 1 hour, followed by centrifugation at $18,000 \mathrm{~g}$ for 20 minutes. The supernatants were then combined with 50 $\mu$ l of Dynabeads protein $G$ (Life Technologies) preincubated with indicated antibodies, followed by rotating for 2 hours or overnight at $4^{\circ} \mathrm{C}$. The protein $G$ was pelleted and washed 3 times using IP buffer or buffer with an additional $500 \mathrm{mM} \mathrm{NaCl}$, followed by 3 washes with PBS, and samples were prepared by addition of $2 \times$ sample loading buffer (Bio$\mathrm{Rad})$. Protein levels in tissue lysates were quantified using a Pierce BCA protein assay kit (Thermo Scientific). For coimmunoprecipitation using mouse brains, tissues were homogenized and prepared in lysis buffer (10 mM Tris-HCl, pH 7.4, 150 mM NaCl, 5 mM EDTA, 0.5\% Nonidet P-40 [vol/vol], 1\% Triton X-100, 0.5\% sodium deoxycholic acid [wt/ vol], Phosphatase Inhibitor Cocktail II and III [Sigma-Aldrich], and complete protease inhibitor mixture) in PBS. IP was then performed as described above. The immunocomplexes were then washed with IP buffer 4 times, and samples were prepared as described above. IB analysis of mouse or human brain samples using total, detergent-soluble, or detergent-insoluble samples was performed as described previously $(23,27)$. Brain tissue lysates or immunoprecipitated samples were electrophoresed on SDS-PAGE gels and transferred to nitrocellulose membranes. Membranes were blocked with $5 \%$ nonfat dry milk (wt/vol) in TBS-T and incubated with primary antibodies. After HRP-conjugated secondary antibody incubation, the IB signal was detected using chemiluminescent substrates (Thermo Scientific).

In vitro kinase assay. In vitro kinase assay was performed with purified recombinant human c-Abl and $\alpha$-synuclein. Proteins were incubated in a kinase assay buffer (20 mM HEPES, pH 7.5, $5 \mathrm{mM}$ EGTA, $20 \mathrm{mM} \beta$-glycerophosphate, $20 \mathrm{mM} \mathrm{MgCl}_{2}, 10 \mu \mathrm{M}$ ATP, and $0.5 \mu \mathrm{Ci}$ of $\gamma^{-32} \mathrm{P}$-ATP at a combined volume of $\left.30 \mu \mathrm{l}\right)$. The reaction mixture was incubated at $30^{\circ} \mathrm{C}$ for 30 minutes. Reactions were quenched by the addition of SDS-sample loading buffer, heated to $70^{\circ} \mathrm{C}$ for 10 minutes, and then loaded onto $4 \%-20 \%$ polyacrylamide gels for SDS-PAGE. Following electrophoresis, gel was transferred to PVDF membrane, heat-sealed in hybridization bags, and exposed to a phosphoimaging screen overnight at room temperature for assessing radioactive ${ }^{32} \mathrm{P}$ incorporation.

2-DE gel electrophoresis and IB analysis. Recombinant nonphosphorylated $\alpha$-synuclein or phosphorylated $\alpha$-synuclein by c-Abl was subjected to 2-DE gel electrophoresis followed by immunoblotting exactly as described previously (23). Briefly, following sample suspension in appropriate buffers, followed by concentration and desalting, samples were applied on immobilized $\mathrm{pH} 4-7$ nonlinear gradient strips. Strips were then equilibrated in appropriate equilibration buffers and subsequently loaded on $9 \%-16 \%$ gradient SDS gels for second-dimensional separation. Immediately after the seconddimension run, gels were transferred to PVDF membrane and anti$\alpha$-synuclein or anti-phosphotyrosine antibody was applied for 2-DE immunoblotting. The corresponding phosphospot in Coomassiestained 2-DE gel was used for identification of the phosphorylation site via tandem mass spectrometry.

Phosphorylation analysis by liquid chromatography-tandem mass spectrometry. Excised 2-DE spots were subjected to a modified in-gel trypsin digestion procedure exactly as described previously (23). Peptide sequences (and hence protein identity) was determined by matching of protein or translated nucleotide databases with the acquired fragmentation pattern by the software program Sequest (Thermo Finnigan) (63). The modification of 79.9663 mass units to serine, threonine, and tyrosine was included in the database searches to determine phosphopeptides. Each phosphopeptide that was determined by the Sequest program was also manually inspected to ensure confidence.

Open-field assay. Open-field test was performed by placing of animals into an infrared beam chamber. Activity was monitored using the photobeam Activity System (San Diego Instruments), which provides a grid of infrared beams. The total number of beam breaks over a period of 30 minutes was recorded and analyzed.

Phospho-Y39 $\alpha$-synuclein polyclonal antibody generation. The $\alpha$-synuclein phosphopeptide TKEGVLY(p)VGSKTKEGC was conjugated to $\mathrm{KLH}$ and injected into rabbits for polyclonal antibody generation. Phospho-Y39 $\alpha$-synuclein antibody was purified from crude sera by affinity depletion and purification.

Statistics. Comparisons between 2 groups were performed with 2-tailed unpaired Student's $t$ tests, and those between more than 2 groups were performed with 1-way ANOVA with Tukey's post-test of multiple comparisons. A $P$ value less than 0.05 was considered statistically significant. Statistical analysis for the survival curves was performed by the Mann-Whitney-Wilcoxon test.

Study approval. All procedures involving animals were approved by and conformed to the guidelines of the Institutional Animal Care Committee of Johns Hopkins University. The Johns Hopkins Medical Institution Joint Committee on Clinical Investigations decided that the studies in this proposal involving human postmortem brains were exempt from human subjects approval because of Federal Register 46.101 exemption number 4 .

\section{Author contributions}

VLD, HSK, and TMD supervised the project. SB, VLD, HSK, and TMD formulated the hypothesis. SB, VLD, HSK, and TMD designed experiments, analyzed data, and wrote the manuscript. SB, PG, SHL, DK, SSK, MK, XM, and YL performed experiments. $\mathrm{OP}$ and JCT provided human postmortem tissues.

\section{Acknowledgments}

This work was supported by grants from the National Institute of Neurological Disorders and Stroke (NINDS, NIH) (NS38377, the Morris K. Udall Parkinson's Disease Research Center, and NS082205) and the JPB Foundation. P. Ge was supported by a Parkinson's Disease Foundation Summer Student Fellowship (PDF-SFW-1572). T.M. Dawson is the Leonard and Madlyn Abramson Professor in Neurodegenerative Diseases. The authors acknowledge the joint participation by the Adrienne Helis Malvin Medical Research Foundation and the Diana Helis Henry Medi- 
cal Research Foundation through their direct engagement in the continuous active conduct of medical research in conjunction with Johns Hopkins Hospital and the Johns Hopkins University School of Medicine and the foundations' Parkinson's Disease Programs, $\mathrm{H}-1$ and $\mathrm{M}-2014$.

Address correspondence to: Han Seok Ko, Neuroregeneration and Stem Cell Programs, Institute for Cell Engineering, Johns Hopkins University School of Medicine, 733 North Broadway, Suite 751A, Baltimore, Maryland 21205, USA. Phone: 410.502.5454; E-mail: hko3@jhmi.edu. Or to: Ted Dawson, Neuroregeneration and Stem Cell Programs, Institute for Cell Engineering, Johns Hopkins University School of Medicine, 733 North Broadway, Suite 731, Baltimore, Maryland 21205, USA. Phone: 410.614.3359; E-mail: tdawson@jhmi.edu.

Yunjong Lee's present address is: Division of Pharmacology, Department of Molecular Cell Biology, Sungkyunkwan University School of Medicine, Samsung Biomedical Research Institute, Suwon, South Korea.
1. Savitt JM, Dawson VL, Dawson TM. Diagnosis and treatment of Parkinson disease: molecules to medicine. J Clin Invest. 2006;116(7):1744-1754.

2. Dauer W, Przedborski S. Parkinson's disease: mechanisms and models. Neuron. 2003;39(6):889-909.

3. Bonnet AM, Jutras MF, Czernecki V, Corvol JC, Vidailhet M. Nonmotor symptoms in Parkinson's disease in 2012: relevant clinical aspects. Parkinsons Dis. 2012;2012:198316.

4. Goedert M. $\alpha$-Synuclein and neurodegenerative diseases. Nat Rev Neurosci. 2001;2(7):492-501.

5. Goedert M, Spillantini MG, Del Tredici K, Braak H. 100 years of Lewy pathology. Nat Rev Neurol. 2013;9(1):13-24.

6. Lee VM, Trojanowski JQ. Mechanisms of Parkinson's disease linked to pathological alpha-synuclein: new targets for drug discovery. Neuron. 2006;52(1):33-38.

7. Dawson TM, Ko HS, Dawson VL. Genetic animal models of Parkinson's disease. Neuron. 2010;66(5):646-661.

8. Maries E, Dass B, Collier TJ, Kordower JH, Steece-Collier K. The role of alpha-synuclein in Parkinson's disease: insights from animal models. Nat Rev Neurosci. 2003;4(9):727-738.

9. Polymeropoulos $\mathrm{MH}$, et al. Mutation in the $\alpha$-synuclein gene identified in families with Parkinson's disease. Science. 1997;276(5321):2045-2047.

10. Zarranz JJ, et al. The new mutation, E46K, of $\alpha$-synuclein causes Parkinson and Lewy body dementia. Ann Neurol. 2004;55(2):164-173.

11. Krüger R, et al. Ala30Pro mutation in the gene encoding $\alpha$-synuclein in Parkinson's disease. Nat Genet. 1998;18(2):106-108.

12. Martin I, Dawson VL, Dawson TM. Recent advances in the genetics of Parkinson's disease. Annu Rev Genomics Hum Genet. 2011;12:301-325.

13. Bezard E, Yue Z, Kirik D, Spillantini MG. Animal models of Parkinson's disease: limits and relevance to neuroprotection studies. Mov Disord. 2013;28(1):61-70.

14. Chesselet MF, Richter F. Modelling of Parkinson's disease in mice. Lancet Neurol. 2011;10(12):1108-1118.

15. Deng H, Yuan L. Genetic variants and animal models in SNCA and Parkinson disease. Ageing Res Rev. 2014;15:161-176.

16. Fernagut PO, Chesselet MF. $\alpha$-Synuclein and transgenic mouse models. Neurobiol Dis. 2004;17(2):123-130.

17. Lee Y, Dawson VL, Dawson TM. Animal models of Parkinson's disease: vertebrate genetics. Cold Spring Harb Perspect Med. 2012;2(10):a009324.
18. Fujiwara H, et al. $\alpha$-Synuclein is phosphorylated in synucleinopathy lesions. Nat Cell Biol. 2002;4(2):160-164.

19. Gao HM, Kotzbauer PT, Uryu K, Leight S, Trojanowski JQ, Lee VM. Neuroinflammation and oxidation/nitration of $\alpha$-synuclein linked to dopaminergic neurodegeneration. J Neurosci. 2008;28(30):7687-7698.

20. Hodara R, et al. Functional consequences of $\alpha$-synuclein tyrosine nitration: diminished binding to lipid vesicles and increased fibril formation. J Biol Chem. 2004;279(46):47746-47753.

21. Giasson BI, et al. Oxidative damage linked to neurodegeneration by selective $\alpha$-synuclein nitration in synucleinopathy lesions. Science. 2000;290(5493):985-989.

22. Hantschel O, Superti-Furga G. Regulation of the c-Abl and Bcr-Abl tyrosine kinases. Nat Rev Mol Cell Biol. 2004;5(1):33-44.

23. Ko HS, et al. Phosphorylation by the c-Abl protein tyrosine kinase inhibits parkin's ubiquitination and protective function. Proc Natl Acad Sci US A. 2010;107(38):16691-16696.

24. Imam SZ, et al. Novel regulation of parkin function through c-Abl-mediated tyrosine phosphorylation: implications for Parkinson's disease. J Neurosci. 2011;31(1):157-163.

25. Hebron ML, Lonskaya I, Moussa CE. Nilotinib reverses loss of dopamine neurons and improves motor behavior via autophagic degradation of $\alpha$-synuclein in Parkinson's disease models. Hum Mol Genet. 2013;22(16):3315-3328.

26. Karuppagounder SS, Brahmachari S, Lee Y, Dawson VL, Dawson TM, Ko HS. The c-Abl inhibitor, nilotinib, protects dopaminergic neurons in a preclinical animal model of Parkinson's disease. Sci Rep. 2014;4:4874.

27. Lee MK, et al. Human $\alpha$-synuclein-harboring familial Parkinson's disease-linked Ala-53 --> Thr mutation causes neurodegenerative disease with alpha-synuclein aggregation in transgenic mice. Proc Natl Acad Sci U S A. 2002;99(13):8968-8973.

28. Martin LJ, et al. Parkinson's disease $\alpha$-synuclein transgenic mice develop neuronal mitochondrial degeneration and cell death. JNeurosci. 2006;26(1):41-50.

29. Colla E, Jensen PH, Pletnikova O, Troncoso JC, Glabe C, Lee MK. Accumulation of toxic $\alpha$-synuclein oligomer within endoplasmic reticulum occurs in $\alpha$-synucleinopathy in vivo. J Neurosci. 2012;32(10):3301-3305

30. Giasson BI, Duda JE, Quinn SM, Zhang B, Trojanowski JQ, Lee VM. Neuronal alpha-synucle- inopathy with severe movement disorder in mice expressing A53T human $\alpha$-synuclein. Neuron. 2002;34(4):521-533.

31. Shockett P, Difilippantonio M, Hellman N, Schatz DG. A modified tetracycline-regulated system provides autoregulatory, inducible gene expression in cultured cells and transgenic mice. Proc Natl Acad Sci U S A. 1995;92(14):6522-6526.

32. Smith WW, et al. Synphilin-1 attenuates neuronal degeneration in the A53T $\alpha$-synuclein transgenic mouse model. Hum Mol Genet. 2010;19(11):2087-2098.

33. Von Coelln R, et al. Loss of locus coeruleus neurons and reduced startle in parkin null mice. Proc Natl Acad Sci U S A. 2004;101(29):10744-10749.

34. von Coelln R, et al. Inclusion body formation and neurodegeneration are parkin independent in a mouse model of $\alpha$-synucleinopathy. J Neurosci. 2006;26(14):3685-3696.

35. Schwartzberg PL, et al. Mice homozygous for the ablm1 mutation show poor viability and depletion of selected B and T cell populations. Cell. 1991;65(7):1165-1175.

36. Tybulewicz VL, Crawford CE, Jackson PK, Bronson RT, Mulligan RC. Neonatal lethality and lymphopenia in mice with a homozygous disruption of the c-abl proto-oncogene. Cell. 1991;65(7):1153-1163.

37. Moresco EM, Donaldson S, Williamson A, Koleske AJ. Integrin-mediated dendrite branch maintenance requires Abelson (Abl) family kinases. J Neurosci. 2005;25(26):6105-6118.

38. Bétemps D, et al. $\alpha$-Synuclein spreading in M83 mice brain revealed by detection of pathological $\alpha$-synuclein by enhanced ELISA. Acta Neuropathol Commun. 2014;2:29.

39. Cunnington R, Iansek R, Johnson KA, Bradshaw JL. Movement-related potentials in Parkinson's disease. Motor imagery and movement preparation. Brain. 1997;120 (pt 8):1339-1353.

40. Burré J, Sharma M, Südhof TC. Systematic mutagenesis of $\alpha$-synuclein reveals distinct sequence requirements for physiological and pathological activities. J Neurosci. 2012;32(43):15227-15242.

41. Schmid AW, Fauvet B, Moniatte M, Lashuel HA. $\alpha$-Synuclein post-translational modifications as potential biomarkers for Parkinson disease and other synucleinopathies. Mol Cell Proteomics. 2013;12(12):3543-3558.

42. Sato H, Kato T, Arawaka S. The role of Ser129 phosphorylation of $\alpha$-synuclein in neurodegeneration of Parkinson's disease: a review of in vivo models. Rev Neurosci. 2013;24(2):115-123.

43. Lue LF, et al. Biochemical increase in phosphor- 
ylated $\alpha$-synuclein precedes histopathology of Lewy-type synucleinopathies. Brain Pathol. 2012;22(6):745-756.

44. Wang Y, et al. Phosphorylated $\alpha$-synuclein in Parkinson's disease. Sci Transl Med. 2012;4(121):121ra20.

45. Gao L, et al. Cerebrospinal fluid $\alpha$-synuclein as a biomarker for Parkinson's disease diagnosis: a systematic review and meta-analysis. Int J Neurosci. 2015;125(9):645-654.

46. Mahul-Mellier AL, et al. c-Abl phosphorylates $\alpha$-synuclein and regulates its degradation: implication for $\alpha$-synuclein clearance and contribution to the pathogenesis of Parkinson's disease. Hum Mol Genet. 2014;23(11):2858-2879.

47. Gaki GS, Papavassiliou AG. Oxidative stressinduced signaling pathways implicated in the pathogenesis of Parkinson's disease. Neuromolecular Med. 2014;16(2):217-230.

48. Imam SZ, et al. Neuroprotective efficacy of a new brain-penetrating C-Abl inhibitor in a murine Parkinson's disease model. PLoS One. 2013;8(5):e65129.

49. Dawson TM, Dawson VL. Parkin plays a role in sporadic Parkinson's disease. Neurodegener Dis. 2014;13(2-3):69-71.

50. Hebron ML, Lonskaya I, Moussa CE. Tyro- sine kinase inhibition facilitates autophagic SNCA $/ \alpha$-synuclein clearance. Autophagy. 2013;9(8):1249-1250.

51. Hebron ML, Lonskaya I, Olopade P, Selby ST, Pagan F, Moussa CE. Tyrosine kinase inhibition regulates early systemic immune changes and modulates the neuroimmune response in $\alpha$-synucleinopathy. JClin Cell Immunol. 2014;5:259.

52. Wu R, et al. c-Abl-p38 $\alpha$ signaling plays an important role in MPTP-induced neuronal death. Cell Death Differ. 2016;23(3):542-552.

53. Tanabe A, Yamamura Y, Kasahara J, Morigaki R, Kaji R, Goto S. A novel tyrosine kinase inhibitor AMN107 (nilotinib) normalizes striatal motor behaviors in a mouse model of Parkinson's disease. Front Cell Neurosci. 2014;8:50.

54. Manley PW, et al. Extended kinase profile and properties of the protein kinase inhibitor nilotinib. Biochim Biophys Acta. 2010;1804(3):445-453.

55. Konig H, et al. Enhanced BCR-ABL kinase inhibition does not result in increased inhibition of downstream signaling pathways or increased growth suppression in CML progenitors. Leukemia. 2008;22(4):748-755.

56. Lee Y, et al. Parthanatos mediates AIMP2-activated age-dependent dopaminergic neuronal loss. Nat Neurosci. 2013;16(10):1392-1400.
57. Smith WW, et al. $\alpha$-Synuclein phosphorylation enhances eosinophilic cytoplasmic inclusion formation in SH-SY5Y cells. J Neurosci. 2005;25(23):5544-5552.

58. Shin JH, et al. PARIS (ZNF746) repression of PGC-1 $\alpha$ contributes to neurodegeneration in Parkinson's disease. Cell. 2011;144(5):689-702.

59. Dauer W, et al. Resistance of $\alpha$-synuclein null mice to the parkinsonian neurotoxin MPTP. Proc Natl Acad Sci U S A. 2002;99(22):14524-14529.

60. Hunot S, et al. JNK-mediated induction of cyclooxygenase 2 is required for neurodegeneration in a mouse model of Parkinson's disease. Proc Natl Acad Sci U S A. 2004;101(2):665-670.

61. Luk KC, et al. Pathological $\alpha$-synuclein transmission initiates Parkinson-like neurodegeneration in nontransgenic mice. Science. 2012;338(6109):949-953.

62. Lane EL, Winkler C, Brundin P, Cenci MA. The impact of graft size on the development of dyskinesia following intrastriatal grafting of embryonic dopamine neurons in the rat. Neurobiol Dis. 2006;22(2):334-345.

63. Griffin PR, MacCoss MJ, Eng JK, Blevins RA, Aaronson JS, Yates JR. Direct database searching with MALDI-PSD spectra of peptides. Rapid Commun Mass Spectrom. 1995;9(15):1546-1551. 